\title{
ON $q$-ANALOGUES OF MULTIPLE ZETA VALUES
}

\author{
JOHANNES SINGER
}

Abstract: We study a $q$-analogue of multiple zeta values that was proposed by Zudilin and is closely related to that of Schlesinger. We explore the double $q$-shuffle structure and provide an Euler decomposition formula. Furthermore we compare our results with the classical multiple zeta values and the $q$-models of Ohno-Okuda-Zudilin and Bradley.

Keywords: multiple zeta values, $q$-analogues, double shuffle relation, Euler decomposition formula.

\section{Introduction}

The classical multiple zeta values (MZVs) are multidimensional generalizations of the Riemann zeta function which are defined for $k_{1}, \ldots, k_{n} \in \mathbb{N}$ with $k_{1}>1$ by the iterated sums

$$
\zeta\left(k_{1}, \ldots, k_{n}\right):=\sum_{m_{1}>\cdots>m_{n}>0} \frac{1}{m_{1}^{k_{1}} \cdots m_{n}^{k_{n}}} .
$$

The first appearance could be traced back to Euler. For instance, he proved the identity $\zeta(2,1)=\zeta(3)$. The systematic study of MZVs was initiated by Hoffman [10] and Zagier [18]. The $\mathbb{Q}$-vector space spanned by

$$
\mathcal{M}:=\left\langle\zeta\left(k_{1}, \ldots, k_{n}\right): k_{1}, \ldots, k_{n} \in \mathbb{N}, k_{1}>1, n \in \mathbb{N}\right\rangle_{\mathbb{Q}}
$$

is an algebra with two different products - the shuffle and the quasi shuffle product that do not coincide. Therefore there are many $\mathbb{Q}$-linear relations involving MZVs. We remember that the quasi shuffle product relies on the series representation (1) of MZVs. The shuffle product is induced from the integral representation of MZVs $([18])$

$$
\zeta\left(k_{1}, \ldots, k_{n}\right)=\int_{1>t_{1}>\cdots>t_{k}>0} \omega_{1}\left(t_{1}\right) \cdots \omega_{k}\left(t_{k}\right),
$$

2010 Mathematics Subject Classification: primary: 11M32 
where $k:=k_{1}+\cdots+k_{n}$ and $\omega_{i}(t):=d t /(1-t)$ if $i \in\left\{k_{1}, k_{1}+k_{2}, \ldots, k_{1}+\right.$ $\left.\cdots+k_{n}\right\}$ and $\omega_{i}(t):=d t / t$ otherwise. In [12] Ihara, Kaneko and Zagier introduced a regularization procedure for the double shuffle relation and there is the conjecture that all $\mathbb{Q}$-linear relations of MZVs can be obtained by this extended double shuffle relation.

In this paper we study a $q$-analog of MZVs $(q$-MZVs). Let $0<q<1$ be a fixed real number. For $\mathbf{k}:=\left(k_{1}, \ldots, k_{n}\right) \in \mathbb{N}_{0}^{n}$ with $k_{1}>0$ we consider the model

$$
\zeta_{q}[\mathbf{k}]:=\sum_{m_{1}>\cdots>m_{n}>0} \frac{q^{k_{1} m_{1}+\cdots+k_{n} m_{n}}}{\left[m_{1}\right]_{q}^{k_{1}} \cdots\left[m_{n}\right]_{q}^{k_{n}}}
$$

where $[m]_{q}:=\frac{1-q^{m}}{1-q}$. Additionally, we regard the $q$-analog of multiple zeta star values $(q$-MZSVs) given by

$$
\zeta_{q}^{\star}[\mathbf{k}]:=\sum_{m_{1} \geqslant \cdots \geqslant m_{n}>0} \frac{q^{k_{1} m_{1}+\cdots+k_{n} m_{n}}}{\left[m_{1}\right]_{q}^{k_{1}} \cdots\left[m_{n}\right]_{q}^{k_{n}}} .
$$

The iterated sums (2) and (3) converge for $k_{1}, \ldots, k_{n} \in \mathbb{N}_{0}$ with $k_{1}>0$ (see [19, Proposition 2.2]). If $\left(k_{1}, \ldots, k_{n}\right) \in \mathbb{N}^{n}$ with $k_{1}>1$, then the $q$-MZVs reduce to the classical MZVs in the limit $q \uparrow 1$. In [20] Zudilin proposed model (2) as an appropriate $q$-extension of MZVs. It essentially coincides with that of Schlesinger in [15]. For technical reasons we regard the extended model given by

$$
\bar{\zeta}_{q}[\mathbf{k}]:=(1-q)^{-|\mathbf{k}|} \zeta_{q}[\mathbf{k}]
$$

On the one hand, model (4) has a very natural quasi $q$-shuffle product which coincides with that of classical MZVs. On the other hand, there was no suitable $q$-shuffle product and hence a lack of $\mathbb{Q}$-linear relations in the algebra

$$
\mathcal{M}_{q}:=\left\langle\bar{\zeta}_{q}\left[k_{1}, \ldots, k_{n}\right]: k_{1}, \ldots, k_{n} \in \mathbb{N}_{0}, k_{1}>0, n \in \mathbb{N}\right\rangle_{\mathbb{Q}}
$$

In this paper we propose a $q$-shuffle product (Theorem 3.2) that is not compatible with the quasi $q$-shuffle product (Theorem 3.3). Therefore we can establish double $q$-shuffle relations for that model (Theorem 3.4). The key ingredient is a representation of (4) via an iteration of Rota-Baxter operators (Proposition 2.6). This progress considerably relies on the techniques of $[4,3]$ where Castillo Medina, Ebrahimi-Fard and Manchon studied the model

$$
\overline{\mathfrak{z}}_{q}[\mathbf{k}]:=\sum_{m_{1}>\cdots>m_{n}>0} \frac{q^{m_{1}}}{\left(1-q^{m_{1}}\right)^{k_{1}} \cdots\left(1-q^{m_{n}}\right)^{k_{n}}}
$$

for $\mathbf{k}:=\left(k_{1}, \ldots, k_{n}\right) \in \mathbb{N}^{n}$ with $k_{1}>1$ which was first proposed by Ohno, Okuda 
and Zudilin in [13]. Moreover the most studied $q$-analogue in literature is that of Bradley (see [2]) given by

$$
\bar{\zeta}_{q}^{B}[\mathbf{k}]:=\sum_{m_{1}>\cdots>m_{n}>0} \frac{q^{\left(k_{1}-1\right) m_{1}+\cdots+\left(k_{n}-1\right) m_{n}}}{\left(1-q^{m_{1}}\right)^{k_{1}} \cdots\left(1-q^{m_{n}}\right)^{k_{n}}}
$$

for $\mathbf{k}:=\left(k_{1}, \ldots, k_{n}\right) \in \mathbb{N}^{n}$ with $k_{1}>1$.

The Rota-Baxter operator characterization of model (4) allows us - as in [4] to find an Euler decomposition formula (Theorem 5.2) that relies on an identity of Rota-Baxter operators (Theorem A.2). Furthermore we generalise this identity to a specific class of two different Rota-Baxter operators (Theorem 5.5) which allows us to present a completely combinatorial proof of Euler's decomposition formula for model (6) (Corollary 5.6). In [1] Bradley has already proved this formula. However he used a differential identity. By mathematical folklore it is well known that Euler's formula relies on the shuffle product. Therefore, in the $q$-case we can interpret the Rota-Baxter operator characterization as a substitute of the integral representation for classical MZVs. The $q$-shuffle product induced by the iteration of Rota-Baxter operators for Bradley's model is addressed in the forthcoming paper [16].

Finally, we show that period-1 sums of the models (2) and (3) completely reduce to a polynomial equation in $q$-ZVs over $\mathbb{Q}$ (Corollary 6.3). For this reason we study polynomials that arise form the well known Spitzer identity for RotaBaxter operators (Theorem 6.2). To translate this result in the $q$-MZ(S)Vs setting, we use the concept of free Rota-Baxter algebras. In order to apply this techniques, we employ a Rota-Baxter operator description of the models (2) and (3) that is different from that regarded in Section 2 and is related to the quasi $q$-shuffle product.

The paper is organized as follows. In Section 2 we introduce the concept of Rota-Baxter algebras and review the construction of free Rota-Baxter algebras and their connection to $q-\mathrm{MZ}(\mathrm{S}) \mathrm{V}$. Furthermore we provide several examples of Rota-Baxter operators and present characterizations of the models given above via iterations of these operators. Section 3 is devoted to the algebraic theory of $\mathbb{Q}$-linear relations of model (4). We establish a double $q$-shuffle product on the algebra $\mathcal{M}_{q}$. Furthermore we illuminate the differential algebra structure of $\mathcal{M}_{q}$ in Section 4. Euler's decomposition formulas for the models (4) and (6) are provided in Section 5. In Section 6 we show that period-1 sums of $q-\mathrm{MZ}(\mathrm{S}) \mathrm{V}$ can be written as certain polynomials in $q-\mathrm{ZV}$. In the Appendix A we just recall the main results of [5] used in our article because the paper has a problem in the correct display of its results.

Notation. We write $\mathbb{N}:=\{1,2,3 \ldots\}$ for the positive integers and $\mathbb{N}_{0}:=\{0,1,2, \ldots\}$ for the additive monoid. The binomial coefficient is defined for any $k, n \in \mathbb{N}_{0}$ with $k \leqslant n$ by $\left(\begin{array}{l}n \\ k\end{array}\right):=\frac{n !}{k !(n-k) !}$. Furthermore we declare the common convention $\left(\begin{array}{l}n \\ k\end{array}\right):=0$ if $k>n$. Further the multinomial coefficient is given for $k_{1}, \ldots, k_{n} \in \mathbb{N}_{0}$ and $k:=k_{1}+\cdots+k_{n}$ by $\left(\begin{array}{c}k \\ k_{1}, \ldots, k_{n}\end{array}\right):=\frac{k !}{k_{1} ! \cdots k_{n} !}$. For an index 
$\mathbf{k}:=\left(k_{1}, \ldots, k_{n}\right) \in \mathbb{N}_{0}^{n}$ we call $n$ the depth of $\mathbf{k}$ and denote by $|\mathbf{k}|:=k_{1}+\cdots+k_{n}$ the weight and by $\operatorname{ht}(\mathbf{k}):=\#\left\{i \in\{1, \ldots, n\}: k_{i}>0\right\}$ the height of $\mathbf{k}$. The symmetric group on $m \in \mathbb{N}$ symbols is denoted by $S_{m}$.

\section{Rota-Baxter algebras and $q$-multiple zeta values}

\section{1. (Free) Rota-Baxter algebras and mixable shuffle}

Let $k$ be a ring, $\lambda \in k$ and $\mathcal{A}$ a $k$-algebra. A Rota-Baxter operator of weight $\lambda$ on $\mathcal{A}$ over $k$ is a $k$-module endomorphism $L$ of $\mathcal{A}$ such that

$$
L(x) L(y)=L(x L(y))+L(L(x) y)+\lambda L(x y)
$$

for any $x, y \in \mathcal{A}$. A Rota-Baxter $k$-algebra of weight $\lambda$ is a pair $(\mathcal{A}, L)$ with a $k$-algebra $\mathcal{A}$ and a Rota-Baxter operator $L$ of weight $\lambda$ on $\mathcal{A}$ over $k$. Let $\left(L_{1}, \mathcal{A}_{1}\right)$ and $\left(L_{2}, \mathcal{A}_{2}\right)$ be two Rota-Baxter $k$-algebras of weight $\lambda$. A homomorphism of Rota-Baxter $k$-algebras $f:\left(L_{1}, \mathcal{A}_{1}\right) \rightarrow\left(L_{2}, \mathcal{A}_{2}\right)$ is a homomorphism $f: \mathcal{A}_{1} \rightarrow \mathcal{A}_{2}$ of $k$-algebras satisfying $f \circ L_{1}=L_{2} \circ f$ on $\mathcal{A}_{1}$.

We review the construction of the free Rota-Baxter algebra of a given unitary $k$-algebra $\mathcal{A}$ (for details see $[8,9]$ ). Let $n \in \mathbb{N}_{0}$. Then we denote by $\mathcal{A}^{\otimes n}$ the $n$-fold tensor product of $\mathcal{A}$ in the category of $k$-modules with the convention $\mathcal{A}^{\otimes 0}=k$. For $n, m \in \mathbb{N}$ we define the set of $(n, m)$-shuffles by

$$
S_{n, m}=\left\{\sigma \in S_{n+m}: \sigma^{-1}(1)<\cdots<\sigma^{-1}(n), \sigma^{-1}(n+1)<\cdots<\sigma^{-1}(m+n)\right\} .
$$

Let $\sigma \in S_{n, m}$. A pair of indices $(k, k+1)$ with $1 \leqslant k<m+n$ is called admissible for $\sigma$ if $\sigma(k) \leqslant n<\sigma(k+1)$. The set of admissible indices for $\sigma$ is denoted by $T_{\sigma}$. We denote by

$$
\bar{S}_{n, m}:=\left\{(\sigma, T): \sigma \in S_{n, m}, T \subseteq T_{\sigma}\right\}
$$

the set of mixable shuffles. Let $x:=x_{1} \otimes \cdots \otimes x_{n} \in \mathcal{A}^{\otimes n}$ and $y:=y_{1} \otimes \cdots \otimes y_{m} \in$ $\mathcal{A}^{\otimes m}$ for $n, m \in \mathbb{N}$. We denote by $x \otimes y=x_{1} \otimes \cdots \otimes x_{n} \otimes y_{1} \otimes \cdots \otimes y_{m} \in \mathcal{A}^{\otimes(n+m)}$ and for $\sigma \in S_{n, m}$

$$
\sigma(x \otimes y)=z_{\sigma(1)} \otimes z_{\sigma(2)} \otimes \cdots \otimes z_{\sigma(n+m)}
$$

where

$$
z_{k}:= \begin{cases}x_{k} & k \in\{1, \ldots, n\} \\ y_{k-n} & k \in\{n+1, \ldots, m+n\} .\end{cases}
$$

Let $\sigma \in S_{n, m}$. For $T \subseteq T_{\sigma}$ we call

$$
\sigma(x \otimes y ; T):=z_{\sigma(1)} \hat{\otimes} \cdots \hat{\otimes} z_{\sigma(n+m)}
$$

the mixable shuffle of $x$ and $y$, where for each pair $(k, k+1), 1 \leqslant k<n+m$,

$$
z_{\sigma(k)} \hat{\otimes} z_{\sigma(k+1)}:= \begin{cases}z_{\sigma(k)} z_{\sigma(k+1)} & (k, k+1) \in T ; \\ z_{\sigma(k)} \otimes z_{\sigma(k+1)} & (k, k+1) \notin T .\end{cases}
$$


Example 2.1. Let $\sigma_{1}:=\left(\begin{array}{ccc}1 & 2 & 3 \\ 1 & 2 & 3\end{array}\right), \sigma_{2}:=\left(\begin{array}{ccc}1 & 2 & 3 \\ 2 & 1 & 3\end{array}\right), x:=x_{1}$ and $y:=$ $y_{1} \otimes y_{2}$. Then we have

$$
\sigma_{1}(x \otimes y ;(1,2))=x_{1} y_{1} \otimes y_{2}, \quad \sigma_{2}(x \otimes y ;(2,3))=y_{1} \otimes x_{1} y_{2} .
$$

The binary operation $\diamond^{+}$given by

$$
x \diamond^{+} y:=\sum_{(\sigma, T) \in \bar{S}_{n, m}} \lambda^{|T|} \sigma(x \otimes y ; T) \in \bigoplus_{j=0}^{n+m} \mathcal{A}^{\otimes j}
$$

can be extended by $k$-linearity and additivity to the $k$-bilinear map

$$
\diamond^{+}: \varpi_{k}^{+}(\mathcal{A}) \times \varpi_{k}^{+}(\mathcal{A}) \rightarrow \varpi_{k}^{+}(\mathcal{A})
$$

in which

$$
\Psi_{k}^{+}(\mathcal{A}):=\bigoplus_{j \in \mathbb{N}_{0}} \mathcal{A}^{\otimes j}
$$

This binary operation is called mixable shuffle product of weight $\lambda$.

Now we construct the free Rota-Baxter algebra on a unitary $k$-algebra $\mathcal{A}$. As

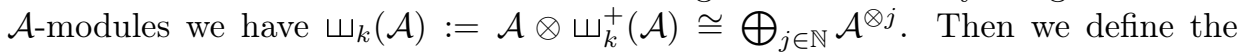
product $\diamond$ on $\varpi_{k}(\mathcal{A})$ via

$$
\begin{aligned}
& \left(x_{0} \otimes x_{1} \otimes \cdots \otimes x_{n}\right) \diamond\left(y_{0} \otimes y_{1} \otimes \cdots \otimes y_{m}\right) \\
& :=\left(x_{0} y_{0}\right) \otimes\left(\left(x_{1} \otimes \cdots \otimes x_{n}\right) \diamond^{+}\left(y_{1} \otimes \cdots \otimes y_{m}\right)\right) \in \mathcal{A}^{\otimes(n+m+1)} .
\end{aligned}
$$

Further we establish the $k$-linear endomorphism $P_{\mathcal{A}}$ on $\varpi_{k}(\mathcal{A})$ by

$$
P_{\mathcal{A}}\left(x_{0} \otimes x_{1} \otimes \cdots \otimes x_{n}\right)=1_{\mathcal{A}} \otimes x_{0} \otimes x_{1} \otimes \cdots \otimes x_{n}
$$

and extending by additivity. Further let $j_{\mathcal{A}}: \mathcal{A} \rightarrow \varpi_{k}(\mathcal{A})$ be the canonical inclusion map.

Theorem 2.2. [8, Theorem 4.1] Let $\mathcal{A}$ be a unitary $k$-algebra. Then the $k$-module $\varpi_{k}(\mathcal{A})$ with the multiplication $\diamond$ is a unitary commutative $k$-algebra. Furthermore the pair $\left(\varpi_{k}(\mathcal{A}), P_{\mathcal{A}}\right)$ with $j_{\mathcal{A}}: \mathcal{A} \rightarrow \varpi_{k}(\mathcal{A})$ is a free Rota-Baxter algebra on $\mathcal{A}$ of weight $\lambda$, i. e. for any Rota-Baxter $k$-algebra $(\mathcal{R}, P)$ of weight $\lambda$ and any $k$-algebra homomorphism $\varphi: \mathcal{A} \rightarrow \mathcal{R}$ there exists a unique Rota-Baxter $k$-algebra homomorphism $\hat{\varphi}:\left(\varpi_{k}(\mathcal{A}), P_{\mathcal{A}}\right) \rightarrow(\mathcal{R}, P)$ such that the following diagram commutes:

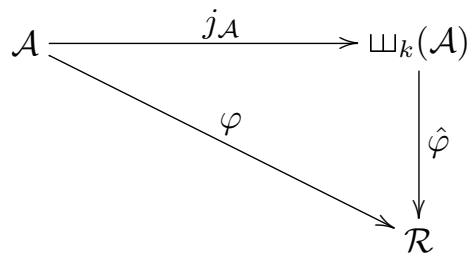


Now we recall the concept of MZV algebras introduced in [6]. Let $\mathcal{R}$ be a $k$-algebra and $P: \mathcal{R} \rightarrow \mathcal{R}$ a partially defined map. If $P$ satisfies the Rota-Baxter identity we call it partially defined Rota-Baxter operator. For $\mathbf{f}:=\left(f_{1}, \ldots, f_{n}\right) \in$ $\mathcal{R}^{n}$ we define the symbol $P_{\mathbf{f}}:=P\left[f_{1}\left[P\left[f_{2} \ldots P\left[f_{n}\right] \ldots\right]\right]\right]$. Let $\mathcal{A}$ be a filtered subalgebra of $\mathcal{R}$. The corresponding ideals with respect to the filtration are denoted by $\mathcal{A}_{k}, k \geqslant 0$. Then $\mathcal{A}$ is called iteratively summable of level $k$ if the formal symbols $P_{\mathrm{f}}$ are well-defined for all $f_{i} \in \mathcal{A}(i \geqslant 1)$ with $f_{n} \in \mathcal{A}_{k}$. For this algebra we call

$$
\mathfrak{A}_{k}:=\left\{P_{\left(f_{1}, \ldots, f_{n}\right)}: f_{i} \in \mathcal{A}, i=1, \ldots, n, f_{n} \in \mathcal{A}_{k}\right\} .
$$

the $M Z V$ algebra of level $k$.

Now let $\mathcal{A}$ be a filtered $k$-algebra. The MZV algebra of level $k$ generated by $\mathcal{A}$ in $\varpi_{k}(\mathcal{A})$ is the subspace $\mathfrak{M}(\mathcal{A})_{k}$ of $\varpi_{k}(\mathcal{A})$ generated by the pure tensors of the form $1 \otimes a_{1} \otimes \cdots \otimes a_{n} \in 1 \otimes \mathcal{A}^{\otimes n}$ with $a_{n} \in \mathcal{A}_{k}$. We call $\mathfrak{M}(\mathcal{A})_{k}$ the universal $M Z V$ algebra of level $k$ generated by $\mathcal{A}$.

Theorem 2.3. [6, Theorem 3.3] Let $\mathcal{R}$ be a $k$-algebra with a partially defined RotaBaxter operator $P$ and let $k \in \mathbb{N}_{0}$. Let $\mathcal{A}$ be an iteratedly summable subalgebra of $\mathcal{R}$ of level $k$. Then we have:

(i) The $M Z V$ algebra $\mathfrak{A}_{k}$ is a subalgebra of $\mathcal{R}$.

(ii) The universal $M Z V$ algebra $\mathfrak{M}(\mathcal{A})_{k}$ is a subalgebra of $\amalg(\mathcal{A})_{k}$.

(iii) There is an algebra surjection

$$
\mathfrak{P}_{k}: \mathfrak{M}(\mathcal{A})_{k} \rightarrow \mathfrak{A}_{k}, \quad 1 \otimes f_{1} \otimes \cdots \otimes f_{n} \mapsto P_{\left(f_{1}, \ldots, f_{n}\right)}
$$

(iv) For an algebra homomorphism $\nu: \mathcal{A} \rightarrow k$ (evaluation) we obtain an algebra homomorphism $\nu \circ \mathfrak{P}_{k}: \mathfrak{M}(\mathcal{A})_{k} \rightarrow k$.

Therefore all algebraic relation among elements $f_{i}, i \in\{1, \ldots, n\}$ in $\mathfrak{M}(\mathcal{A})_{k}$ for a fixed $k$ can be "transformed" into algebraic relations of the MZV algebra $\mathfrak{A}_{k}$ via $\mathfrak{P}_{k}$ or to $k$ via $\nu \circ \mathfrak{P}_{k}$ (see $[6$, Corollary 3.4$]$ ). We use this result in Section 6 .

\subsection{Examples of Rota-Baxter algebras}

Now we provide several well known examples of Rota-Baxter algebras which are related to MZVs and their $q$-analogues (see e.g. [4], [3] and for further examples [7]).

Example 2.4. Let $\mathcal{A}_{1}$ be the $\mathbb{R}$-algebra of continuous functions on $\mathbb{R}$. Due to the integration by parts formula the integration operator $I: \mathcal{A}_{1} \rightarrow \mathcal{A}_{1}$

$$
I[f](x):=\int_{0}^{x} f(t) d t \quad(x \in \mathbb{R})
$$

is a Rota-Baxter operator of weight 0 . 
For a formal power series $f \in \mathbb{Q}[[t]]$ we define the $q$-dilation operator as

$$
E_{q}[f](t):=f(q t),
$$

where $0<q<1$. Let $\mathcal{A}:=t \mathbb{Q}[[t, q]]$ be the space of formal power series in the two variables $t$ and $q$ without an term of degree zero in $t$. We can interpret $\mathcal{A}$ as the $\mathbb{Q}[[q]]$-algebra $t \mathbb{Q}[[t]]$. Then the $\mathbb{Q}[[q]]$-linear map $P_{q}: \mathcal{A} \rightarrow \mathcal{A}$ is defined by

$$
P_{q}[f](t):=\sum_{n>0} E_{q}^{n}[f](t)
$$

On the same algebra $\mathcal{A}$ we define the linear operator $\bar{P}_{q}: \mathcal{A} \rightarrow \mathcal{A}$ given by

$$
\bar{P}_{q}[f](t):=\sum_{n \geqslant 0} E_{q}^{n}[f](t) .
$$

We have the following result:

Proposition 2.5. Let $0<q<1$ and $\mathcal{A}$ the $\mathbb{Q}[[q]]$-algebra $t \mathbb{Q}[[t]]$.

(i) The pair $\left(\mathcal{A}, P_{q}\right)$ is a Rota-Baxter $\mathbb{Q}[[q]]$-algebra of weight 1 , i. e.

$$
P_{q}[f] P_{q}[g]=P_{q}\left[P_{q}[f] g\right]+P_{q}\left[f P_{q}[g]\right]+P_{q}[f g]
$$

for any $f, g \in \mathcal{A}$.

(ii) The pair $\left(\mathcal{A}, \bar{P}_{q}\right)$ is a Rota-Baxter $\mathbb{Q}[[q]]$-algebra of weight-1, i. e.

$$
\bar{P}_{q}[f] \bar{P}_{q}[g]=\bar{P}_{q}\left[\bar{P}_{q}[f] g\right]+\bar{P}_{q}\left[f \bar{P}_{q}[g]\right]-\bar{P}_{q}[f g]
$$

for any $f, g \in \mathcal{A}$.

(iii) For the mixed product we have

$$
P_{q}[f] \bar{P}_{q}[g]=\bar{P}_{q}\left[P_{q}[f] g\right]+P_{q}\left[f \bar{P}_{q}[g]\right]
$$

for $f, g \in \mathcal{A}$.

Proof. (i) and (ii) can be found in [4]. For (iii) we have

$$
\begin{aligned}
P_{q}[f](t) \bar{P}_{q}[g](t) & =\sum_{n>0} f\left(q^{n} t\right) \sum_{m \geqslant 0} g\left(q^{m} t\right) \\
& =\sum_{n>m \geqslant 0} f\left(q^{n} t\right) g\left(q^{m} t\right)+\sum_{m \geqslant n>0} f\left(q^{n} t\right) g\left(q^{m} t\right) \\
& =\sum_{n>0, m \geqslant 0} f\left(q^{n+m} t\right) g\left(q^{m} t\right)+\sum_{m \geqslant 0, n>0} f\left(q^{n} t\right) g\left(q^{m+n} t\right) \\
& =\bar{P}_{q}\left[P_{q}[f] g\right](t)+P_{q}\left[f \bar{P}_{q}[g]\right](t)
\end{aligned}
$$

for any $f, g \in \mathcal{A}$. 


\subsection{Models arising from the iteration of Rota-Baxter operators}

In this section we provide Rota-Baxter operator identities for the extended versions of the models regarded in the introduction.

Proposition 2.6. Let $0<q<1$ and $n \in \mathbb{N}$. With the operators $P_{q}$ and $\bar{P}_{q}$ defined in (7) and (8) and $y(t):=\frac{t}{1-t} \in t \mathbb{Q}[[t]]$ we have

(i) for $\mathbf{k}:=\left(k_{1}, \ldots, k_{n}\right) \in \mathbb{N}_{0}^{n}$ with $k_{1}>0$

$$
\bar{\zeta}_{q}[\mathbf{k}]=P_{q}^{k_{1}}\left[y P_{q}^{k_{2}}\left[y \cdots P_{q}^{k_{n}}[y] \cdots\right]\right](1) ;
$$

(ii) for $\mathbf{k}:=\left(k_{1}, \ldots, k_{n}\right) \in \mathbb{N}^{n}$ with $k_{1}>1$

$$
\overline{\mathfrak{z}}_{q}[\mathbf{k}]=\bar{P}_{q}^{k_{1}}\left[y \bar{P}_{q}^{k_{2}}\left[y \cdots \bar{P}_{q}^{k_{n}}[y] \cdots\right]\right](q) ;
$$

(iii) for $\mathbf{k}:=\left(k_{1}, \ldots, k_{n}\right) \in \mathbb{N}^{n}$ with $k_{1}>1$

$$
\bar{\zeta}_{q}^{B}[\mathbf{k}]=P_{q}^{k_{1}-1} \bar{P}_{q}\left[y P_{q}^{k_{2}-1} \bar{P}_{q}\left[y \cdots P_{q}^{k_{n}-1} \bar{P}_{q}[y] \cdots\right]\right](1) .
$$

Remark 2.7. In [4, Eq. (20)] the authors provide a characterization of $\zeta_{q}^{B}$ via the iteration of the Rota-Baxter operator $P_{q}$ and a set of power series in $\mathbb{Q}[[t, q]]$ whose cardinality is equal to the length of $\mathbf{k}$. Heuristically, they work with an algebra which is induced by infinitely many letters. In contrast to this our description in Proposition 2.6 (iii) relies only on the two Rota-Baxter operators $P_{q}$ and $\bar{P}_{q}$ as well as on $y \in t \mathbb{Q}[[t]]$. Therefore the underlying algebra is induced by only three letters. The reduction to three letters is in accordance with the double shuffle relation of Takeyama in [17].

Proof of Proposition 2.6. (ii) was proved in [3, Eq. (17)]. (i) follows by induction on the height of $\mathbf{k}$. Let $\mathbf{k}:=\left(k_{1}, \ldots, k_{n}\right) \in \mathbb{N}_{0}^{n}$ with $k_{1}>0$ and fixed $n \in \mathbb{N}$. For $\operatorname{ht}(\mathbf{k})=1$ the index is of the form $\mathbf{k}=\left(k_{1}, 0, \ldots, 0\right)$ with $k_{1}>0$. Then we have

$$
\begin{aligned}
P_{q}^{k_{1}}\left[y^{n}\right](t) & =P_{q}^{k_{1}-1}\left[\sum_{m>0}\left(\frac{q^{m^{m}} t}{1-q^{m} t}\right)^{n}\right]=P_{q}^{k_{1}-1}\left[\sum_{m>0} \prod_{i=1}^{n} \sum_{l_{i}>0} q^{m l_{i}} t^{l_{i}}\right] \\
& =P_{q}^{k_{1}-1}\left[\sum_{\substack{l_{i}>0 \\
i=1, \ldots, n}} t^{l_{1}+\cdots+l_{n}} \frac{q^{l_{1}+\cdots+l_{n}}}{1-q^{l_{1}+\cdots+l_{n}}}\right] \\
& =P_{q}^{k_{1}-1}\left[\sum_{m_{1}>\cdots>m_{n}>0} t^{m_{1}} \frac{q^{m_{1}}}{1-q^{m_{1}}}\right] \\
& =\sum_{m_{1}>\cdots>m_{n}>0} t^{m_{1}} \frac{q^{k_{1} m_{1}}}{\left(1-q^{m_{1}}\right)^{k_{1}}}
\end{aligned}
$$

and evaluation at $t=1$ shows that the base case is true. By the induction hypothesis, (i) is true for $\operatorname{ht}(\mathbf{k}):=s \in \mathbb{N}$. Then in the inductive step $s \rightarrow s+1$ we 
assume that $\mathrm{ht}(\mathbf{k})=s+1$ and therefore $\mathbf{k}=\left(\mathbf{k}^{\prime}, \mathbf{k}^{\prime \prime}\right)$ with $\mathbf{k}^{\prime}:=\left(k_{1}^{\prime}, 0, \ldots, 0\right) \in \mathbb{N}^{a}$ $(1 \leqslant a \leqslant n-1)$ and $\mathbf{k}^{\prime \prime}:=\left(k_{a+1}^{\prime \prime}, \ldots, k_{n}^{\prime \prime}\right) \in \mathbb{N}^{(n-a)}$ such that ht $\left(\mathbf{k}^{\prime}\right)=1$ and ht $\left(\mathbf{k}^{\prime \prime}\right)=s$. Then we obtain

$$
\begin{aligned}
& P_{q}^{k_{1}}\left[y P_{q}^{k_{2}}\left[y \cdots P_{q}^{k_{n}}[y] \cdots\right]\right](t) \\
& =P_{q}^{k_{1}^{\prime}}\left[y^{a}(t) \sum_{m_{a+1}>\cdots>m_{n}>0} t^{m_{a+1}} \frac{q^{k_{a+1}^{\prime \prime} m_{a+1}+\cdots+k_{n}^{\prime \prime} m_{n}}}{\left(1-q^{m_{a+1}}\right)^{k_{a+1}^{\prime \prime}} \ldots\left(1-q^{m_{n}}\right)^{k_{n}^{\prime \prime}}}\right] \\
& =P_{q}^{k_{1}^{\prime}-1}\left[\sum_{m>0}\left(\frac{q^{m} t}{1-q^{m} t}\right)^{a} \sum_{m_{a+1}>\cdots>m_{n}>0} q^{m m_{a+1}} t^{m_{a+1}}\right. \\
& \left.\times \frac{q^{k_{a+1}^{\prime \prime} m_{a+1}+\cdots+k_{n}^{\prime \prime} m_{n}}}{\left(1-q^{m_{a+1}}\right)^{k_{a+1}^{\prime \prime}} \ldots\left(1-q^{m_{n}}\right)^{k_{n}^{\prime \prime}}}\right] \\
& =P_{q}^{k_{1}^{\prime}-1}\left[\sum_{\substack{l_{i}>0 \\
i=1, \ldots, a}} \sum_{m_{a+1}>\cdots>m_{n}>0} t^{l_{1}+\cdots+l_{a}+m_{a+1}} \frac{q^{l_{1}+\cdots+l_{a}+m_{a+1}}}{1-q^{l_{1}+\cdots+l_{a}+m_{a+1}}}\right. \\
& \left.\times \frac{q^{k_{a+1}^{\prime \prime} m_{a+1}+\cdots+k_{n}^{\prime \prime} m_{n}}}{\left(1-q^{m_{a+1}}\right)^{k_{a+1}^{\prime \prime}} \cdots\left(1-q^{m_{n}}\right)^{k_{n}^{\prime \prime}}}\right] \\
& =P_{q}^{k_{1}^{\prime}-1}\left[\sum_{m_{1}>\cdots>m_{a}>m_{a+1}>\cdots>m_{n}>0} t^{m_{1}} \frac{q^{m_{1}}}{1-q^{m_{1}}} \frac{q^{k_{a+1}^{\prime \prime} m_{a+1}+\cdots+k_{n}^{\prime \prime} m_{n}}}{\left(1-q^{m_{a+1}}\right)^{k_{a+1}^{\prime \prime}} \ldots\left(1-q^{m_{n}}\right)^{k_{n}^{\prime \prime}}}\right] \\
& =\sum_{m_{1}>\cdots>m_{a}>m_{a+1}>\cdots>m_{n}>0} t^{m_{1}} \frac{q^{k_{1}^{\prime} m_{1}}}{\left(1-q^{m_{1}}\right)^{k_{1}^{\prime}}} \frac{q^{k_{a+1}^{\prime \prime} m_{a+1}+\cdots+k_{n}^{\prime \prime} m_{n}}}{\left(1-q^{m_{a+1}}\right)^{k_{a+1}^{\prime \prime}} \cdots\left(1-q^{m_{n}}\right)^{k_{n}^{\prime \prime}}} .
\end{aligned}
$$

Now we evaluate at $t=1$ and get the claim. (iii) follows by a similar induction on the shifted height of $\mathbf{k}:=\left(k_{1}, \ldots, k_{n}\right)$ which is defined by $\tilde{\mathrm{ht}}(\mathbf{k}):=\#\{i \in$ $\left.\{1, \ldots, n\}: k_{i}>1\right\}$.

In $[4$, Corollary 5$]$ it was shown that

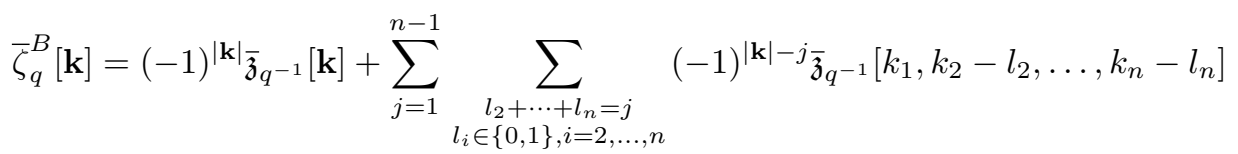

for $\mathbf{k}:=\left(k_{1}, \ldots, k_{n}\right) \in \mathbb{N}^{n}$ with $k_{1}>1$. In the next lemma we disclose the connection between the models (4) and (5).

Lemma 2.8. Let $n \in \mathbb{N}$. For $\mathbf{k}:=\left(k_{1}, \ldots, k_{n}\right) \in \mathbb{N}_{0}^{n}$ with $k_{1}>0$ we have

$$
\overline{\mathfrak{z}}_{q}[\mathbf{k}]=\sum_{r_{1}=1}^{k_{1}} \sum_{r_{2}=0}^{k_{2}} \cdots \sum_{r_{n}=0}^{k_{n}}\left(\begin{array}{l}
k_{1}-1 \\
r_{1}-1
\end{array}\right) \prod_{j=2}^{n}\left(\begin{array}{l}
k_{j} \\
r_{j}
\end{array}\right) \bar{\zeta}_{q}\left[r_{1}, \ldots, r_{n}\right] .
$$


Proof. The shift operator $\mathcal{S}_{j}: \mathcal{M}_{q} \rightarrow \mathcal{M}_{q}$ is defined as

$$
\mathcal{S}_{j} \bar{\zeta}_{q}\left[k_{1}, \ldots, k_{n}\right]:=\bar{\zeta}_{q}\left[k_{1}, \ldots, k_{n}\right]+\bar{\zeta}_{q}\left[k_{1}, \ldots, k_{j-1}, k_{j}-1, k_{j+1}, \ldots, k_{n}\right]
$$

for $j \in\{1, \ldots, n\}$. Obviously, for $s_{1}, \ldots, s_{n} \in \mathbb{N}_{0}$ with $s_{1} \leqslant k_{1}-1$ and $s_{j} \leqslant k_{j}$ for $j \neq 1$ we have

$$
\mathcal{S}_{1}^{s_{1}} \circ \cdots \circ \mathcal{S}_{n}^{s_{n}} \bar{\zeta}_{q}\left[k_{1}, \ldots, k_{n}\right]=\sum_{r_{1}=0}^{s_{1}} \cdots \sum_{r_{n}=0}^{s_{n}}\left(\prod_{j=1}^{n}\left(\begin{array}{c}
s_{j} \\
r_{j}
\end{array}\right) \bar{\zeta}_{q}\left[k_{1}-r_{1}, \ldots, k_{n}-r_{n}\right]\right)
$$

by the definition of the shift operator. On the other hand, we see that

$$
\begin{aligned}
& \mathcal{S}_{n} \bar{\zeta}_{q}\left[k_{1}, \cdots, k_{n}\right] \\
= & \bar{\zeta}_{q}\left[k_{1}, \ldots, k_{n}\right]+\bar{\zeta}_{q}\left[k_{1}, \ldots, k_{n-1}, k_{n}-1\right] \\
= & \sum_{m_{1}>\cdots>m_{n}>0} \frac{q^{k_{1} m_{1}+\cdots+k_{n-1} m_{n-1}}}{\left(1-q^{m_{1}}\right)^{k_{1}} \ldots\left(1-q^{m_{n-1}}\right)^{k_{n-1}}}\left(\frac{q^{k_{n} m_{n}}}{\left(1-q^{m_{n}}\right)^{k_{n}}}+\frac{q^{\left(k_{n}-1\right) m_{n}}}{\left(1-q^{m_{n}}\right)^{k_{n}-1}}\right) \\
= & \sum_{m_{1}>\cdots>m_{n}>0} \frac{q^{k_{1} m_{1}+\cdots+k_{n-1} m_{n-1}+\left(k_{n}-1\right) m_{n}}}{\left(1-q^{m_{1}}\right)^{k_{1}} \ldots\left(1-q^{m_{n}}\right)^{k_{n}}} .
\end{aligned}
$$

Therefore we easily get by induction that

$$
\mathcal{S}_{1}^{s_{1}} \circ \cdots \circ \mathcal{S}_{n}^{s_{n}} \bar{\zeta}_{q}\left[k_{1}, \ldots, k_{n}\right]=\sum_{m_{1}>\cdots>m_{d}>0} \frac{q^{\left(k_{1}-s_{1}\right) m_{1}+\cdots+\left(k_{n}-s_{n}\right) m_{n}}}{\left(1-q^{m_{1}}\right)^{k_{1}} \ldots\left(1-q^{m_{n}}\right)^{k_{n}}}
$$

(cf. [19, proof of Proposition 2.5]). We choose $s_{1}=k_{1}-1$ and $s_{j}=k_{j}$ for $j \in\{2, \ldots, n\}$ to complete the proof.

\section{Algebraic theory of $\mathbb{Q}$-linear relations}

This section is devoted to the study of the $\mathbb{Q}$-linear relations for the model $\bar{\zeta}_{q}$ that are induced by a double $q$-shuffle product.

\section{1. $q$-shuffle}

Let $X:=\{y, p\}$ be the set of letters and $X^{*}$ be the free monoid of $X$ with respect to concatenation. The elements of $X^{*}$ are called words and the empty word in $X^{*}$ is denoted by $\mathbf{1}$. The $q$-shuffle product $\amalg: X^{*} \times X^{*} \rightarrow \mathbb{Q}\langle X\rangle$ is defined iteratively by

(i) $\mathbf{1} \amalg v=v ш \mathbf{1}=v$ for any $v \in X^{*}$;

(ii) $(y u) \amalg v=u \amalg(y v)=y(u \amalg v)$ for any $u, v \in X^{*}$;

(iii) $(p u) \amalg(p v)=p(u \amalg(p v))+p((p u) \amalg v)+p(u \amalg v)$ for any $u, v \in X^{*}$.

Now we can extent the $q$-shuffle product to $\mathbb{Q}\langle X\rangle$ by distributivity.

Lemma 3.1. The q-shuffle product $\amalg$ is commutative and associative. 
Proof. We define the length $l(u)$ of the word $u \in X^{*}$ as the number of letters of $u$. Now let $v, w \in X^{*}$. We prove commutativity by induction on the length $l(v)+l(w)$. The base case is obvious due to (i). By induction hypothesis we get:

Case 1: $v$ or $w$ begin with $y$. Without loss of generality let $v=y v^{\prime}\left(v^{\prime} \in X^{*}\right)$ with $l\left(v^{\prime}\right)=l(v)-1$ :

$$
v ш w=y\left(v^{\prime} ш w\right)=y\left(w \varpi v^{\prime}\right)=w ш v
$$

Case 2: $v$ and $w$ begin with $p$. Therefore we have $v=p v^{\prime}$ and $w=p w^{\prime}$ $\left(v^{\prime}, w^{\prime} \in X^{*}\right)$ with $l\left(v^{\prime}\right)=l(v)-1$ and $l\left(w^{\prime}\right)=l(w)-1$ :

$$
v ш w=p\left(v^{\prime} ш p w^{\prime}\right)+p\left(p v^{\prime} \amalg w^{\prime}\right)+p\left(v^{\prime} ш w^{\prime}\right)=w ш v
$$

Now we prove associativity by induction on the length $l(u)+l(v)+l(w)$ for $u, v, w \in$ $X^{*}$. The base case is clear. The induction hypothesis implies:

Case 1: $u, v$ or $w$ begin with $y$. Without loss of generality let $u=y u^{\prime}\left(u^{\prime} \in X^{*}\right)$ with $l\left(u^{\prime}\right)=l(u)-1$ :

$$
\begin{aligned}
(u \uplus v) \amalg w & =\left(y\left(u^{\prime} \amalg v\right) \amalg w\right)=y\left(\left(u^{\prime} \amalg v\right) \amalg w\right)=y\left(u^{\prime} \amalg(v ш w)\right) \\
& =u \amalg(v ш w)
\end{aligned}
$$

Case 2: $u, v$ and $w$ begin with $p$. Therefore we have $u=p u^{\prime}, v=p v^{\prime}$ and $w=p w^{\prime}\left(u^{\prime}, v^{\prime}, w^{\prime} \in X^{*}\right)$ with $l\left(u^{\prime}\right)=l(u)-1, l\left(v^{\prime}\right)=l(v)-1$ and $l\left(w^{\prime}\right)=l(w)-1$ :

$$
\begin{aligned}
& (u \amalg v) \amalg w=\left(p u^{\prime} \amalg p v^{\prime}\right) \amalg p w^{\prime}=p\left\{\left(u^{\prime} \amalg p v^{\prime}\right) \amalg p w^{\prime}+p\left(u^{\prime} \amalg p v^{\prime}\right) \amalg w^{\prime}\right. \\
& +\left(u^{\prime} \amalg p v^{\prime}\right) \amalg w^{\prime}+\left(p u^{\prime} \amalg v^{\prime}\right) \amalg p w^{\prime} \\
& +p\left(p u^{\prime} \amalg v^{\prime}\right) \amalg w^{\prime}+\left(p u^{\prime} ш v^{\prime}\right) \amalg w^{\prime} \\
& +\left(u^{\prime} ш v^{\prime}\right) \amalg p w^{\prime}+p\left(u^{\prime} \amalg v^{\prime}\right) \amalg w^{\prime} \\
& \left.+\left(u^{\prime} \amalg v^{\prime}\right) \amalg w^{\prime}\right\} \\
& =p\left\{u^{\prime} \amalg p\left(v^{\prime} \amalg p w^{\prime}\right)+p u^{\prime} \amalg\left(v^{\prime} \amalg p w^{\prime}\right)\right. \\
& +u^{\prime} \amalg\left(v^{\prime} \amalg p w^{\prime}\right)+u^{\prime} \amalg p\left(p v^{\prime} \amalg w^{\prime}\right) \\
& +p u^{\prime} ш\left(p v^{\prime} \amalg w^{\prime}\right)+u^{\prime} \amalg\left(p v^{\prime} \amalg w^{\prime}\right) \\
& +u^{\prime} \amalg p\left(v^{\prime} \amalg w^{\prime}\right)+p u^{\prime} \amalg\left(v^{\prime} \amalg w^{\prime}\right) \\
& \left.+u^{\prime} ш\left(v^{\prime} \amalg w^{\prime}\right)\right\} \\
& =p u^{\prime} \amalg\left(p v^{\prime} \amalg p w^{\prime}\right)=u \amalg(v ш w)
\end{aligned}
$$

This completes the proof.

The set $Y:=p X^{*} y$ consists of the words beginning with $p$ and ending with $y$. This generates the subalgebra $\langle Y\rangle_{\mathbb{Q}}$ of $\mathbb{Q}\langle X\rangle$ given by

$$
\langle Y\rangle_{\mathbb{Q}}=\mathbb{Q} \mathbf{1}+p \mathbb{Q}\langle X\rangle y
$$

Now we define the $\operatorname{map} \bar{\zeta}_{q}^{\varpi}: Y \rightarrow \mathbb{R}$ by

$$
\bar{\zeta}_{q}^{\uplus}\left[p^{k_{1}} y \cdots p^{k_{n}} y\right]:=\bar{\zeta}_{q}\left[k_{1}, \ldots, k_{n}\right]
$$

and extend it via $\bar{\zeta}_{q}^{\amalg}[\mathbf{1}]:=1$ and $\mathbb{Q}$-linearity to $\langle Y\rangle_{\mathbb{Q}}$. 
Now we have the following result:

Theorem 3.2. The map

$$
\bar{\zeta}_{q}^{\uplus}:\left(\langle Y\rangle_{\mathbb{Q}}, ш\right) \rightarrow(\mathbb{Q}[[q]], \cdot)
$$

is a homomorphism of commutative algebras. Especially we have

$$
\bar{\zeta}_{q}^{\uplus}[u] \bar{\zeta}_{q}^{\varpi}[v]=\bar{\zeta}_{q}^{Ш}[u \amalg v]
$$

for all $u, v \in Y$.

Proof. The associativity and commutativity of the shuffle product $\amalg$ is an immediate consequence of Lemma 3.1. Due to relation (iii) in the definition of $\amalg$ and the Rota-Baxter identity for $P_{q}$, the map $\phi_{q}:\langle Y\rangle_{\mathbb{Q}} \rightarrow \mathcal{A}$ given by

$$
p^{k_{1}} y p^{k_{2}} y \cdots p^{k_{n}} y \mapsto P_{q}^{k_{1}}\left[y P_{q}^{k_{2}}\left[y \cdots P_{q}^{k_{n}}[y] \cdots\right]\right]
$$

is an algebra homomorphism. Since

$$
\bar{\zeta}_{q}^{\amalg}[w]=\left.\phi_{q}(w)(t)\right|_{t=1}
$$

for any word $w \in Y$ we obtain the claimed formula.

\subsection{Quasi $q$-shuffle}

Let $U:=\left\{u_{i}: i \in \mathbb{N}_{0}\right\}$ and $U^{*}$ the free monoid of $U$ with respect to concatenation. The empty word is denoted by $\mathbf{1}$. Now we define the quasi $q$-shuffle product $\sqcup: U^{*} \times U^{*} \rightarrow \mathbb{Q}\langle U\rangle$, that coincides with the classical quasi shuffle product, recursively

(i) $u_{s} \sqcup \mathbf{1}=\mathbf{1} \sqcup u_{s}=u_{s}$ for all $s \in \mathbb{N}_{0}$;

(ii) $\left(u_{s} v\right) \sqcup\left(u_{t} w\right)=u_{s}\left(v \sqcup\left(u_{t} w\right)\right)+u_{t}\left(\left(u_{s} v\right) \sqcup w\right)+u_{s+t}(v \sqcup w)$ for all $s, t \in \mathbb{N}_{0}$ and $v, w \in U^{*}$.

Now we extend $\sqcup$ to $\mathbb{Q}\langle U\rangle$ by distributivity. Further we define the set of convergent words by

$$
V:=\left\{u_{k_{1}} \cdots u_{k_{n}} \in U^{*}:\left(k_{1}, \ldots, k_{n}\right) \in \mathbb{N}_{0}^{n}, n \in \mathbb{N}, k_{1}>0\right\}
$$

and denote $\langle V\rangle_{\mathbb{Q}}$ as the subalgebra of $\mathbb{Q}\langle U\rangle$ that is generated by $V$.

The $\operatorname{map} \bar{\zeta}_{q}^{\sqcup}: V \rightarrow \mathbb{R}$ is given by

$$
\bar{\zeta}_{q}^{\sqcup}\left[u_{k_{1}} \cdots u_{k_{n}}\right]:=\bar{\zeta}_{q}\left[k_{1}, \ldots, k_{n}\right]
$$

for words in $V$ and is extended to $\langle V\rangle_{\mathbb{Q}}$ by $\bar{\zeta}_{q}^{\sqcup}[\mathbf{1}]:=1$ and $\mathbb{Q}$-linearity. 
Theorem 3.3. The map

$$
\bar{\zeta}_{q}^{\sqcup}:\left(\langle V\rangle_{\mathbb{Q}}, \sqcup\right) \rightarrow(\mathbb{Q}[[q]], \cdot)
$$

is a homomorphism of commutative algebras. Especially we have

$$
\bar{\zeta}_{q}^{\sqcup}[u] \bar{\zeta}_{q}^{\sqcup}[v]=\bar{\zeta}_{q}^{\sqcup}[u \sqcup v]
$$

for all $u, v \in V$.

Proof. From [11, Theorem 2.1] we know that $\sqcup$ is commutative and associative. The second claim is an obvious consequence of the multiplication of two series of MZVs. One should keep in mind that we just have to substitute the terms $q^{m} /[m]_{q}$ to obtain the classical MZVs.

\subsection{Double $q$-shuffle relation}

Using Theorem 3.2 and 3.3 we can produce $\mathbb{Q}$-linear relations between the $\bar{\zeta}_{q}$. We define the map $\Phi: Y \rightarrow V$ via

$$
p^{k_{1}} y \cdots p^{k_{n}} y \mapsto u_{k_{1}} \cdots u_{k_{n}}
$$

and extend it to the $\mathbb{Q}$-vector spaces that are spanned by $Y$ and $V$. Obviously, the extended map $\Phi:\langle Y\rangle_{\mathbb{Q}} \stackrel{\sim}{\longrightarrow}\langle V\rangle_{\mathbb{Q}}$ is a $\mathbb{Q}$-vector space isomorphism.

Theorem 3.4. For $u, v \in Y$ we have

$$
\bar{\zeta}_{q}^{\sqcup}[\Phi(u) \sqcup \Phi(v)-\Phi(u \amalg v)]=0 .
$$

Proof. For $w:=p^{k_{1}} y \cdots p^{k_{n}} y \in Y$ with $\left(k_{1}, \ldots, k_{n}\right) \in \mathbb{N}_{0}^{n}, k_{1}>0$, we have

$$
\bar{\zeta}_{q}^{\uplus}[w]=\bar{\zeta}_{q}\left[k_{1}, \ldots, k_{n}\right]=\bar{\zeta}_{q}^{\sqcup}[\Phi(w)] .
$$

Therefore Theorem 3.2 and 3.3 imply

$$
\begin{aligned}
\bar{\zeta}_{q}^{\sqcup}[\Phi(u) \sqcup \Phi(v)] & =\bar{\zeta}_{q}^{\sqcup}[\Phi(u)] \bar{\zeta}_{q}^{\sqcup}[\Phi(v)]=\bar{\zeta}_{q}^{\uplus}[u] \bar{\zeta}_{q}^{\uplus}[v] \\
& =\bar{\zeta}_{q}^{\uplus}[u \amalg v]=\bar{\zeta}_{q}^{\sqcup}[\Phi(u \amalg v)]
\end{aligned}
$$

for $u, v \in Y$.

\section{4. q-regularization and classical case}

The $q$-MZVs allow us to deduce identities for classical MZVs. The parameter $q$ acts as an intrinsic regulator. Therefore we need no additional regularization in the sense of Ihara, Kaneko and Zagier. The most prominent relation which is not covered by classical unregulated double shuffle relation is Euler's identity for MZVs given by $\zeta(2,1)=\zeta(3)$. For $p y, p^{2} y \in Y$ we calculate

$$
\Phi(p y) \sqcup \Phi\left(p^{2} y\right)=u_{1} u_{2}+u_{2} u_{1}+u_{3}
$$


and

$$
\Phi\left(p y \amalg p^{2} y\right)=u_{1} u_{2}+2 u_{2} u_{1}+u_{2} u_{0}+u_{1}^{2} .
$$

Therefore we get

$$
\bar{\zeta}_{q}[3]=\bar{\zeta}_{q}[2,1]+\bar{\zeta}_{q}[2,0]+\bar{\zeta}_{q}[1,1]
$$

Multiplying (9) with $(1-q)^{3}$ results in

$$
\zeta_{q}[3]=\zeta_{q}[2,1]+(1-q)\left(\zeta_{q}[2,0]+\zeta_{q}[1,1]\right) .
$$

Since $\lim _{q \uparrow 1}(1-q)\left(\zeta_{q}[2,0]+\zeta_{q}[1,1]\right)=0$ we get Euler's identity in the limit $q \uparrow 1$.

For $p^{2} y \in Y$ we calculate

$$
\Phi\left(p^{2} y\right) \sqcup \Phi\left(p^{2} y\right)=2 u_{2} u_{2}+u_{4}
$$

and

$$
\Phi\left(p^{2} y ш p^{2} y\right)=2 u_{2} u_{2}+4 u_{3} u_{1}+2 u_{3} u_{0}+4 u_{2} u_{1}+u_{2} u_{0} .
$$

Therefore we get

$$
\bar{\zeta}_{q}[4]=4 \bar{\zeta}_{q}[3,1]+2 \bar{\zeta}_{q}[3,0]+4 \bar{\zeta}_{q}[2,1]+\bar{\zeta}_{q}[2,0]
$$

Again, we get the classical identity $\zeta(4)=4 \zeta(3,1)$ in the limit $q \uparrow 1$.

For $p y \in Y$ we calculate

$$
\Phi(p y) \sqcup \Phi(p y)=2 u_{1} u_{1}+u_{2}
$$

and

$$
\Phi(p y \amalg p y)=2 u_{1} u_{1}+u_{1} u_{0} .
$$

Therefore we get

$$
\bar{\zeta}_{q}[2]=\bar{\zeta}_{q}[1,0]
$$

and in the limit case we obtain

$$
\lim _{q \uparrow 1}(1-q) \zeta_{q}[1,0]=\frac{\pi^{2}}{6} .
$$

\section{Differential algebra structure}

We introduce the derivation operator $\delta: \mathbb{Q}[[q]] \rightarrow \mathbb{Q}[[q]]$ given by $\delta:=q \frac{d}{d q}$. The main result of this section is 
Theorem 4.1. The algebra $\mathcal{M}_{q}$ of $q-M Z V s$ is a differential algebra with respect to $\delta$. Especially we have

$$
\begin{aligned}
& \delta \bar{\zeta}_{q}\left[k_{1}, \ldots, k_{n}\right] \\
&= \sum_{r=1}^{n} k_{r}\left\{(n-r+1)\left(\bar{\zeta}_{q}\left[k_{1}, \ldots, k_{n}\right]+\bar{\zeta}_{q}\left[k_{1}, \ldots, k_{r}+1, \ldots, k_{n}\right]\right)\right. \\
&\left.+\sum_{s=r}^{n}\left(\bar{\zeta}_{q}\left[k_{1}, \ldots, k_{s}, 0, k_{s+1}, \ldots, k_{n}\right]+\bar{\zeta}_{q}\left[k_{1}, \ldots, k_{r}+1, \ldots, k_{s}, 0, k_{s+1}, \ldots, k_{n}\right]\right)\right\} \\
& \text { for }\left(k_{1}, \ldots, k_{n}\right) \in \mathbb{N}_{0} \text { with } k_{1}>0 .
\end{aligned}
$$

Proof. It suffices to prove the formula. We get

$$
\begin{aligned}
\delta \bar{\zeta}_{q}\left[k_{1}, \ldots, k_{n}\right]= & \sum_{m_{1}>\cdots>m_{n}>0} \frac{\left(k_{1} m_{1}+\cdots+k_{n} m_{n}\right) q^{k_{1} m_{1}+\cdots+k_{n} m_{n}}}{\left(1-q^{m_{1}}\right)^{k_{1}} \cdots\left(1-q^{m_{n}}\right)^{k_{n}}} \\
& +\sum_{m_{1}>\cdots>m_{n}>0} \sum_{r=1}^{n} \frac{k_{r} m_{r} q^{k_{1} m_{1}+\cdots+\left(k_{r}+1\right) m_{r}+\cdots+k_{n} m_{n}}}{\left(1-q^{m_{1}}\right)^{k_{1}} \cdots\left(1-q^{m_{r}}\right)^{k_{r}+1} \cdots\left(1-q^{m_{n}}\right)^{k_{n}}} \\
= & \sum_{r=1}^{n} k_{r} \sum_{m_{1}>\cdots>m_{n}>0} \frac{m_{r} q^{k_{1} m_{1}+\cdots+k_{n} m_{n}}}{\left(1-q^{m_{1}}\right)^{k_{1}} \cdots\left(1-q^{m_{n}}\right)^{k_{n}}} \\
& +\sum_{r=1}^{n} k_{r} \sum_{m_{1}>\cdots>m_{n}>0} \frac{m_{r} q^{k_{1} m_{1}+\cdots+\left(k_{r}+1\right) m_{r}+\cdots+k_{n} m_{n}}}{\left(1-q^{m_{1}}\right)^{k_{1}} \cdots\left(1-q^{m_{r}}\right)^{k_{r}+1} \cdots\left(1-q^{m_{n}}\right)^{k_{n}}}
\end{aligned}
$$

Now we use

$$
\begin{aligned}
m_{r}= & (n-r+1)+\left(m_{r}-m_{r+1}-1\right) \\
& +\left(m_{r+1}-m_{r+2}-1\right)+\cdots+\left(m_{n-1}-m_{n}-1\right)+\left(m_{n}-1\right) .
\end{aligned}
$$

By summation along the anti-diagonal we get

$$
\sum_{m_{1}>\cdots>m_{n}>0} \frac{\left(m_{s}-m_{s+1}-1\right) q^{k_{1} m_{1}+\cdots+k_{n} m_{n}}}{\left(1-q^{m_{1}}\right)^{k_{1}} \ldots\left(1-q^{m_{n}}\right)^{k_{n}}}=\bar{\zeta}_{q}\left[k_{1}, \ldots, k_{s}, 0, k_{s+1}, \ldots, k_{n}\right] .
$$

Therefore

$$
\begin{aligned}
\delta \bar{\zeta}_{q}\left[k_{1}, \ldots, k_{n}\right] & \sum_{r=1}^{n} k_{r}\left\{(n-r+1) \bar{\zeta}_{q}\left[k_{1}, \ldots, k_{n}\right]+\sum_{s=r}^{n} \bar{\zeta}_{q}\left[k_{1}, \ldots, k_{s}, 0, k_{s+1}, \ldots, k_{n}\right]\right\} \\
& +\sum_{r=1}^{n} k_{r}\left\{(n-r+1) \bar{\zeta}_{q}\left[k_{1}, \ldots, k_{r}+1, \ldots, k_{n}\right]\right. \\
& \left.+\sum_{s=r}^{n} \bar{\zeta}_{q}\left[k_{1}, \ldots, k_{r}+1, \ldots k_{s}, 0, k_{s+1}, \ldots, k_{n}\right]\right\}
\end{aligned}
$$

which yields the claim. 


\section{Euler's decomposition formula}

In the framework of classical MZVs, Euler proved the following decomposition formula

$$
\zeta(a) \zeta(b)=\sum_{l=0}^{a-1}\left(\begin{array}{c}
b+l-1 \\
b-1
\end{array}\right) \zeta(b+l, a-l)+\sum_{l=0}^{b-1}\left(\begin{array}{c}
a+l-1 \\
a-1
\end{array}\right) \zeta(a+l, b-l)
$$

for $a, b \geqslant 2$. This result can be deduced from [5, Theorem 1] (see Theorem A.1) using the Rota-Baxter operator $I$.

For model (4), using the shuffle product induced by the operator $P_{q}$, we get Theorem 5.2. The result relies on the same identity in Rota-Baxter algebras as in [4]. The second part of this section is devoted to Bradley's model. We use Proposition 2.6 (iii) to present a purely combinatorial proof of Euler's decomposition formula (Corollary 5.6) relying on a Rota-Baxter operator identity (Theorem 5.5). Note that the proof of Euler's decomposition formula in [2] relies on a differential identity. Zhao also provided a rather complicated formula in [19] using a $q$-analogue of Jackson's integral formula. In [17] Takeyama proposed an extension of the algebra

$$
\mathcal{M}_{q}^{\mathrm{B}}:=\left\langle\bar{\zeta}_{q}^{B}[\mathbf{k}]: \mathbf{k} \in \mathbb{N}^{n}, k_{1}>1, n \in \mathbb{N}\right\rangle_{\mathbb{Q}}
$$

and proved that there is a double shuffle product. Takeyama's extension also contains the terms $\varphi_{q}[k](k \in \mathbb{N})$. The iteration of Rota-Baxter operators $P$ and $\bar{P}$ as described in Proposition 2.6 can also be used to establish a double shuffle product. This will be discussed in [16].

\subsection{Euler's decomposition formula for Zudilin's model}

We start with the following result:

Proposition 5.1. For $k \in \mathbb{N}$ we have

$$
\bar{\zeta}_{q}[k, 0]=\sum_{m=1}^{k-1} \frac{(-1)^{m+1}}{k-m} \delta \bar{\zeta}_{q}[k-m]-\bar{\zeta}_{q}[k]+(-1)^{k+1}\left(\bar{\zeta}_{q}[1]+\bar{\zeta}_{q}[2]\right) .
$$

Proof. For $k=1$ we have $\bar{\zeta}_{q}[1,0]=\bar{\zeta}_{q}[2]$ which is exactly (10). Now we can conclude from Theorem 4.1 and the induction hypothesis that

$$
\begin{aligned}
\bar{\zeta}_{q}[k+1,0] & =\frac{1}{k} \delta \bar{\zeta}_{q}[k]-\bar{\zeta}_{q}[k]-\bar{\zeta}_{q}[k+1]-\bar{\zeta}_{q}[k, 0] \\
& =\sum_{m=0}^{k-1} \frac{(-1)^{m}}{k-m} \delta \bar{\zeta}_{q}[k-m]-\bar{\zeta}_{q}[k+1]+(-1)^{k+2}\left(\bar{\zeta}_{q}[1]+\bar{\zeta}_{q}[2]\right) \\
& =\sum_{m=1}^{k} \frac{(-1)^{m+1}}{k+1-m} \delta \bar{\zeta}_{q}[k+1-m]-\bar{\zeta}_{q}[k+1]+(-1)^{k+2}\left(\bar{\zeta}_{q}[1]+\bar{\zeta}_{q}[2]\right),
\end{aligned}
$$

which yields the claim. 
Theorem 5.2. For $a, b \in \mathbb{N}$ we have

$$
\begin{aligned}
\bar{\zeta}_{q}[a] \bar{\zeta}_{q}[b]= & \sum_{l=0}^{a-1} \sum_{k=0}^{a-1-l}\left(\begin{array}{c}
l+b-1 \\
b-1
\end{array}\right)\left(\begin{array}{l}
b \\
k
\end{array}\right) \bar{\zeta}_{q}[b+l, a-l-k] \\
& +\sum_{l=0}^{b-1} \sum_{k=0}^{b-1-l}\left(\begin{array}{c}
l+a-1 \\
a-1
\end{array}\right)\left(\begin{array}{l}
a \\
k
\end{array}\right) \bar{\zeta}_{q}[a+l, b-l-k] \\
& +\sum_{l=1}^{a+b-2} \alpha_{l} \delta \bar{\zeta}_{q}[l]+\sum_{l=1}^{\min (a, b)} \beta_{l} \bar{\zeta}[a+b-l]+(-1)^{a+b}\left(\bar{\zeta}_{q}[1]+\bar{\zeta}_{q}[2]\right)
\end{aligned}
$$

where $\alpha_{l}$ and $\beta_{l}$ are coefficients depending on $a$ and $b$ given by

$$
\begin{aligned}
& \alpha_{l}:= \begin{cases}\frac{(-1)^{a+b+l}}{l}, & 1 \leqslant l \leqslant \max (a, b)-1, \\
\frac{(-1)^{a+b+l}}{l} \sum_{j=l-\max (a, b)+1}^{\min (a, b)-1}(-1)^{j-1}\left(\begin{array}{c}
a+b-j-1 \\
a-j, b-j, j-1
\end{array}\right), \quad \text { else; }\end{cases} \\
& \beta_{l}:=-\left(\begin{array}{c}
a+b-l-1 \\
a-l, b-l, l-1
\end{array}\right) .
\end{aligned}
$$

Remark 5.3. If we assume $a, b>1$ and multiply Euler's formula in Theorem 5.2 with $(1-q)^{a+b}$ we obtain immediately the classical formula (9) in the limit $q \uparrow 1$.

Proof. From Theorem A.2 and the proof of Corollary 12 in [4] we observe that the summation corresponding to the domains $D_{1}, D_{2}, D_{3}$ and $D_{4}$ corresponds to

$$
\begin{aligned}
\sum_{l=0}^{a-1} \sum_{k=0}^{a-1-l}\left(\begin{array}{c}
l+b-1 \\
b-1
\end{array}\right)\left(\begin{array}{l}
b \\
k
\end{array}\right) \bar{\zeta}_{q}[b & +l, a-l-k] \\
& +\sum_{l=0}^{b-1} \sum_{k=0}^{b-1-l}\left(\begin{array}{c}
l+a-1 \\
a-1
\end{array}\right)\left(\begin{array}{l}
a \\
k
\end{array}\right) \bar{\zeta}_{q}[a+l, b-l-k] .
\end{aligned}
$$

For the sum over the domain $D_{5}$ we obtain with Proposition 5.1

$$
\begin{aligned}
& s_{a, b}:=\sum_{\substack{j=\max (a, b) \\
\min (a, b)}}^{a+b-1}\left(\begin{array}{c}
j-1 \\
j-b, j-a, a+b-j-1
\end{array}\right) \bar{\zeta}_{q}[j, 0] \\
& =\sum_{j=1}^{\min (a, b)}\left(\begin{array}{c}
a+b-j-1 \\
a-j, b-j, j-1
\end{array}\right) \bar{\zeta}_{q}[a+b-j, 0] \\
& =\sum_{j=1}^{\min (a, b)}\left(\begin{array}{c}
a+b-j-1 \\
a-j, b-j, j-1
\end{array}\right) \sum_{m=1}^{a+b-j-1} \frac{(-1)^{m+1}}{a+b-j-m} \delta \bar{\zeta}_{q}[a+b-j-m] \\
& -\sum_{j=1}^{\min (a, b)}\left(\begin{array}{c}
a+b-j-1 \\
a-j, b-j, j-1
\end{array}\right) \bar{\zeta}_{q}[a+b-j] \\
& +\sum_{j=1}^{\min (a, b)}\left(\begin{array}{c}
a+b-j-1 \\
a-j, b-j, j-1
\end{array}\right)(-1)^{a+b-j-1}\left(\bar{\zeta}_{q}[1]+\bar{\zeta}_{q}[2]\right) .
\end{aligned}
$$


In order to simplify the terms we use the following combinatorial result:

Lemma 5.4. For $a, b \in \mathbb{N}$ we have

$$
\sum_{j=1}^{\min (a, b)}(-1)^{j+1}\left(\begin{array}{c}
a+b-j-1 \\
a-j, b-j, j-1
\end{array}\right)=1
$$

Proof. Without loss of generality we assume $a \leqslant b \in \mathbb{N}$. We prove the claim by induction on $a$. The base case is true because

$$
\left(\begin{array}{c}
b-1 \\
0, b-1,0
\end{array}\right)=1
$$

for all $b \geqslant 1$. In the inductive step let $a+1 \leqslant b$. Then obviously $a \leqslant b$ and we obtain with the induction hypothesis

$$
\begin{aligned}
\sum_{j=1}^{a+1}(-1)^{j+1}\left(\begin{array}{c}
a+b-j \\
a-j+1, b-j, j-1
\end{array}\right) & (-1)^{a}\left(\begin{array}{c}
b-1 \\
0, b-a-1, a
\end{array}\right) \\
& +\sum_{j=1}^{a}(-1)^{j+1} \frac{a+b-j}{a-j+1}\left(\begin{array}{c}
a+b-j-1 \\
a-j, b-j, j-1
\end{array}\right) \\
= & +(-1)^{a}\left(\begin{array}{c}
b-1 \\
0, b-a-1, a
\end{array}\right) \\
& +(b-1) \sum_{j=1}^{a}(-1)^{j+1}\left(\begin{array}{c}
a+b-j-1 \\
a-j+1, b-j, j-1
\end{array}\right) \\
= & 1+(b-1) \sum_{j=1}^{a+1}(-1)^{j+1}\left(\begin{array}{c}
a+b-j-1 \\
a-j+1, b-j, j-1
\end{array}\right) \\
= & 1 .
\end{aligned}
$$

The last equality is true, since we have

$$
\sum_{j=1}^{a+1}(-1)^{j+1}\left(\begin{array}{c}
a+b-j-1 \\
a-j+1, b-j, j-1
\end{array}\right)=\sum_{j=0}^{a} \frac{(-1)^{a-j}}{j+b-1}\left(\begin{array}{c}
j+b-1 \\
j, b-a+j-1, a-j
\end{array}\right)=0
$$

due to [4, Lemma 11]. This completes the proof. 
Therefore we have

$$
\begin{aligned}
& s_{a, b}=\sum_{j=1}^{\min (a, b)}\left(\begin{array}{c}
a+b-j-1 \\
a-j, b-j, j-1
\end{array}\right)(-1)^{a+b-j} \sum_{m=1}^{a+b-j-1} \frac{(-1)^{m+1}}{m} \delta \bar{\zeta}_{q}[m] \\
& -\sum_{j=1}^{\min (a, b)}\left(\begin{array}{c}
a+b-j-1 \\
a-j, b-j, j-1
\end{array}\right) \bar{\zeta}_{q}[a+b-j]+(-1)^{a+b}\left(\bar{\zeta}_{q}[1]+\bar{\zeta}_{q}[2]\right) \\
& =(-1)^{a+b} \sum_{m=1}^{\max (a, b)-1} \frac{(-1)^{m}}{m} \delta \bar{\zeta}_{q}[m] \\
& +\sum_{j=1}^{\min (a, b)}\left(\begin{array}{c}
a+b-j-1 \\
a-j, b-j, j-1
\end{array}\right) \sum_{m=\max (a, b)}^{a+b-j-1} \frac{(-1)^{a+b-j-1+m}}{m} \delta \bar{\zeta}_{q}[m] \\
& +\sum_{j=1}^{\min (a, b)} \beta_{j} \bar{\zeta}_{q}[a+b-j]+(-1)^{a+b}\left(\bar{\zeta}_{q}[1]+\bar{\zeta}_{q}[2]\right) \\
& =(-1)^{a+b} \sum_{m=1}^{\max (a, b)-1} \frac{(-1)^{m}}{m} \delta \bar{\zeta}_{q}[m] \\
& +\sum_{m=\max (a, b)}^{a+b-2} \frac{(-1)^{m}}{m} \delta \bar{\zeta}_{q}[m] \sum_{j=m-\max (a, b)+1}^{\min (a, b)-1}(-1)^{a+b-j-1}\left(\begin{array}{c}
a+b-j-1 \\
a-j, b-j, j-1
\end{array}\right) \\
& +\sum_{j=0}^{a-1} \beta_{j} \bar{\zeta}_{q}[j+b]+(-1)^{a+b}\left(\bar{\zeta}_{q}[1]+\bar{\zeta}_{q}[2]\right) \\
& =\sum_{k=1}^{a+b-2} \alpha_{k} \delta \bar{\zeta}_{q}[k]+\sum_{j=1}^{\min (a, b)-1} \beta_{j} \bar{\zeta}_{q}[a+b-j]+(-1)^{a+b}\left(\bar{\zeta}_{q}[1]+\bar{\zeta}_{q}[2]\right)
\end{aligned}
$$

finishing the proof of Theorem 5.2.

\subsection{Euler's decomposition formula for Bradley's model}

Let $(P, \mathcal{A})$ and $(Q, \mathcal{A})$ be two Rota-Baxter $k$-algebras of weight $\alpha$ respectively $\beta$. We call them compatible, if they satisfy the relations

$$
P[f] Q[g]=P[f Q[g]]+Q[P[f] g]
$$

and

$$
Q[f] P[g]=Q[f P[g]]+P[Q[f] g]
$$

for any $f, g \in \mathcal{A}$. One should note that this is fulfilled by $P$ and $\bar{P}$ as shown in Proposition 2.5. Now we are interested in the operator $T\left(a_{1}, a_{2} ; b_{1}, b_{2} ; c_{1}, c_{2}\right)$ : $\mathcal{A} \times \mathcal{A} \rightarrow \mathcal{A}$ which is defined as

$$
T\left(a_{1}, a_{2} ; b_{1}, b_{2} ; c_{1}, c_{2}\right)=P^{c_{1}} Q^{c_{2}}\left[P^{a_{1}}\left[Q^{a_{2}}[\cdot]\right] \cdot P^{b_{1}}\left[Q^{b_{2}}[\cdot]\right]\right]
$$


for $a_{1}, a_{2}, b_{1}, b_{2}, c_{1}, c_{2} \in \mathbb{N}_{0}$. We have the following result:

Theorem 5.5. Let $(P, \mathcal{A})$ and $(Q, \mathcal{A})$ be two compatible Rota-Baxter $k$-algebras of weight $\alpha$ respectively $\beta$. For $a, b \in \mathbb{N}$ we have

$$
\begin{aligned}
& T(a-1,1 ; b-1,1 ; 0,0) \\
&=\sum_{s=0}^{a-1} \sum_{t=0}^{a-1-s} \alpha^{t}\left(\begin{array}{c}
s+b-1 \\
b-1
\end{array}\right)\left(\begin{array}{c}
b-1 \\
t
\end{array}\right) T(a-s-t-1,1 ; 0,0 ; b+s-1,1) \\
&+\sum_{s=0}^{b-1} \sum_{t=0}^{b-1-s} \alpha^{t}\left(\begin{array}{c}
s+a-1 \\
a-1
\end{array}\right)\left(\begin{array}{c}
a-1 \\
t
\end{array}\right) T(0,0 ; b-s-t-1,1 ; a+s-1,1) \\
&+\beta \sum_{s=1}^{\min (a, b)} \alpha^{s-1}\left(\begin{array}{c}
a+b-s-1 \\
a-s, b-s, s-1
\end{array}\right) T(0,0 ; 0,0 ; a+b-s-1,1) .
\end{aligned}
$$

In order to prove the theorem we use the graphical notation of [5]. A product on $\mathcal{A}$ is represented by

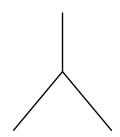

the Rota-Baxter operator $P$ by

$$
\uparrow
$$

and the Rota-Baxter operator $Q$ by

$$
1
$$

We have four calculation rules:

(A) The Rota-Baxter identity of $P$ is decoded by

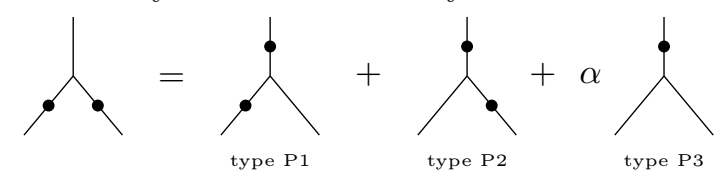

(B) The Rota-Baxter identity of $Q$ is decoded by

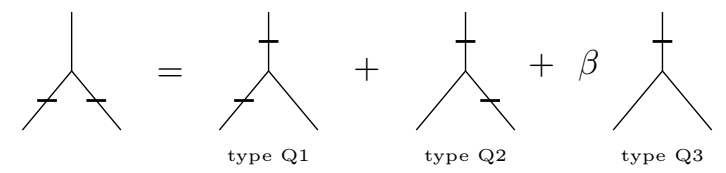


(C) Due to equation (12) the mixed application of the operators $P$ and $Q$ is given by

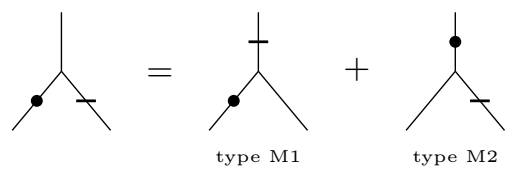

(D) Due to equation (13) the mixed application of the operators $Q$ and $P$ is given by

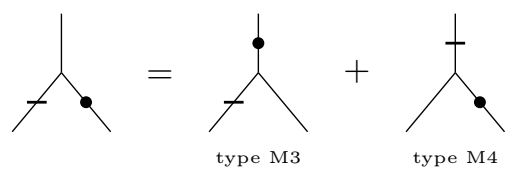

Proof of Theorem 5.5. Let $a, b \in \mathbb{N}$. We regard the operator $T(a-1,1 ; b-$ $1,1 ; 0,0)$ which corresponds to the tree<smiles>CC(C)C(C)C</smiles>

From the iterated applications of the Rota-Baxter identities of $P$ and $Q$ and the interplay of $P$ and $Q$ we conclude that the tree can be written as a $k[\alpha, \beta]$-linear combination of trees exhibiting the following three canonical forms
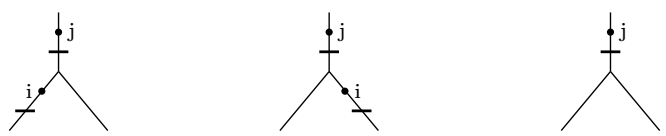

with $i, j \in \mathbb{N}_{0}$ depending on $a$ and $b$.

We begin by counting the occurrence of the canonical form related to $T(i, 1 ; 0,0 ; j, 1)$ (for $i, j \in \mathbb{N}_{0}$ depending on $a, b$ ) which is represented by the following tree:

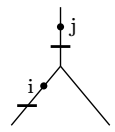

There are $j+1$ applications of our calculation rules (A)-(D). The last application is either of type M1 if $i>0$ or of type Q1 if $i=0$. Therefore we have $j$ applications of our calculation rules (A)-(D) to transform the tree $T(a-1,1 ; b-1,1 ; 0,0)$ to

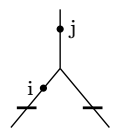


In order to attain this form we have to move $b-1$ dots from the right leg to the neck. Furthermore we can annihilate dots but we can not create new ones. Therefore $b-1 \leqslant j \leqslant a+b-2$. For $i$ we have the obvious restriction $0 \leqslant i \leqslant a-1$. Additionally, we have to ensure $i+j \leqslant a+b-2$. Hence $i \leqslant \min (a-1, a+b-j-2)=$ $a+b-j-2$ since $j \geqslant b-1$. On the other hand the number of annihilated dots is bounded from above by $\min (a, b)-1$. Hence $i+j \geqslant \max (a, b)-1$ and finally

$$
i \geqslant \max (0, \max (a, b)-1-j)=\max (0, a-j-1)
$$

due to $j \geqslant b-1$. Now the $j$ moves of dots rely on $k_{1}$ moves of type $\mathrm{P} 2$ or $\mathrm{M} 2, k_{2}$ moves of type P1 or M3 and $k_{3}$ moves of type P3. Note that there is no intrinsic permutation of $\mathrm{P} 2$ and $\mathrm{M} 2$ in $k_{1}$ because $k_{1}$ counts the number of dots moved from the left leg to the neck. The choice of type P2 or M2 is then determined by the fact of having a dot or a stroke on the right leg. The same argument holds for $k_{2}$. All in all we get the following system of equations

$$
k_{1}+k_{2}+k_{3}=j, \quad k_{1}+k_{3}=a-1-i, \quad k_{2}+k_{3}=b-1 .
$$

Then we obtain

$$
k_{1}=j-b+1, \quad k_{2}=i+j-a+1, \quad k_{3}=a+b-i-j-2 .
$$

Hence the term $T(i, 1 ; 0,0 ; j, 1)$ arises

$$
\left(\begin{array}{c}
j \\
k_{1}, k_{2}, k_{3}
\end{array}\right)=\left(\begin{array}{c}
j \\
j-b+1, i+j-a+1, a+b-i-j-2
\end{array}\right)
$$

times in the the expansion of $T(a-1,1 ; b-1,1 ; 0,0)$. The power of the weight factor $\alpha$ of our canonical form is determined by $k_{3}$. All in all the number of appearances of the canonical form in the expansion of the original operator $T$ is given by

$$
\begin{aligned}
& \sum_{j=b-1}^{a+b-2} \sum_{i=\max (0, a-j-1)}^{a+b-j-2} \alpha^{a+b-i-j-2} \\
& \times\left(\begin{array}{c}
j \\
j-b+1, i+j-a+1, a+b-i-j-2
\end{array}\right) T(i, 1 ; 0,0 ; j, 1) \\
= & \sum_{j=0}^{a-1} \sum_{i=0}^{\min (b-1, a-j-1)} \alpha^{i}\left(\begin{array}{c}
j+b-1 \\
j, b-i-1, i
\end{array}\right) T(a-i-j-1,1 ; 0,0 ; b+j-1,1) \\
= & \sum_{j=0}^{a-1} \sum_{i=0}^{a-j-1} \alpha^{i}\left(\begin{array}{c}
j+b-1 \\
b-1
\end{array}\right)\left(\begin{array}{c}
b-1 \\
i
\end{array}\right) T(a-i-j-1,1 ; 0,0 ; b+j-1,1) .
\end{aligned}
$$

In the second case we count the trees corresponding to $T(0,0 ; i, 1 ; j, 1)$. This is completely analogous to the first case. 
In the last case we count the trees reducing to $T(0,0 ; 0,0 ; j, 1)$. The graphical representation is given by

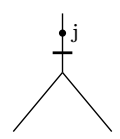

There are $j+1$ moves obeying the calculation rules (A)-(D). The last move must be of type Q3 and gives rise to the weight factor $\beta$. Therefore we have to apply $j$ moves of dots to the tree

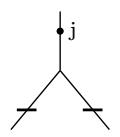

With a similar argumentation as in the first case we deduce $\max (a, b)-1 \leqslant j \leqslant$ $a+b-2$. The $j$ moves of dots rely on $k_{1}$ moves of type $\mathrm{P} 2$ or $\mathrm{M} 2, k_{2}$ moves of type $\mathrm{P} 1$ or $\mathrm{M} 3$ and $k_{3}$ moves of type P3. We get the following system of equations

$$
k_{1}+k_{2}+k_{3}=j, \quad k_{1}+k_{3}=a-1, k_{2}+k_{3}=b-1 .
$$

Therefore we get

$$
k_{1}=j-b+1, \quad k_{2}=j-a+1, \quad k_{3}=a+b-j-2
$$

and the term $T(0,0 ; 0,0 ; j, 1)$ arises

$$
\left(\begin{array}{c}
j \\
j-b+1, j-a+1, a+b-j-2
\end{array}\right)
$$

times in the the expansion of $T(a-1,1 ; b-1,1 ; 0,0)$. This leads to

$$
\begin{array}{r}
\beta \sum_{j=\max (a, b)-1}^{a+b-2} \alpha^{a+b-j-2}\left(\begin{array}{c}
j \\
j-b+1, j-a+1, a+b-j-2
\end{array}\right) T(0,0 ; 0,0 ; j, 1) \\
=\beta \sum_{j=1}^{\min (a, b)} \alpha^{j-1}\left(\begin{array}{c}
a+b-j-1 \\
a-j, b-j, j-1
\end{array}\right) T(0,0 ; 0,0 ; a+b-j-1,1) .
\end{array}
$$

This concludes the proof. 
Using Proposition 2.6, Theorem 5.5 immediately implies the following result:

Corollary 5.6 ([1]). For $a, b \in \mathbb{N}_{\geqslant 2}$ we have

$$
\begin{aligned}
\bar{\zeta}_{q}^{B}[a] \bar{\zeta}_{q}^{B}[b]= & \sum_{s=0}^{a-1} \sum_{t=0}^{a-1-s}\left(\begin{array}{c}
s+b-1 \\
b-1
\end{array}\right)\left(\begin{array}{c}
b-1 \\
t
\end{array}\right) \bar{\zeta}_{q}^{B}[b+s, a-s-t] \\
& +\sum_{s=0}^{b-1} \sum_{t=0}^{b-1-s}\left(\begin{array}{c}
s+a-1 \\
a-1
\end{array}\right)\left(\begin{array}{c}
a-1 \\
t
\end{array}\right) \bar{\zeta}_{q}^{B}[a+s, b-s-t] \\
& -\sum_{s=1}^{\min (a, b)} \frac{(a+b-s-1) !}{(a-s) !(b-s) !(s-1) !} \varphi_{q}[a+b-s],
\end{aligned}
$$

where

$$
\varphi_{q}[k]:=\sum_{n>0} \frac{(n-1) q^{(k-1) n}}{\left(1-q^{n}\right)^{k}}=\sum_{n>0} \frac{n q^{(k-1) n}}{\left(1-q^{n}\right)^{k}}-\bar{\zeta}_{q}^{B}[k] .
$$

\section{Polynomials related to Spitzer's identity}

We start with the well known Spitzer-Identity.

Theorem 6.1 ([14]). Let $\mathcal{A}$ be a unitary commutative $\mathbb{Q}$-algebra and $P$ a RotaBaxter operator of weight $\lambda \in \mathbb{Q}^{\times}$. Then for $a \in \mathcal{A}$ we have

$$
\exp \left(-\sum_{k \geqslant 1} \frac{\lambda^{k-1}(-x)^{k} P\left(a^{k}\right)}{k}\right)=\sum_{n \geqslant 0} x^{n} P_{a}^{n}(1)
$$

in the ring of formal power series $\mathcal{A}[[x]]$ where we used the notation $P_{c}(a)=P(a c)$ and $P_{a}^{n}$ denotes the $n$-fold composition of $P_{a}$.

The set of Rota-Baxter operators has no known algebraic structure. Therefore we cannot apply Spitzer's identity to iterations of Rota-Baxter operators $P_{q}$ and $\bar{P}_{q}$ defined in Section 2.2 because e. g. the composition of two Rota-Baxter operators is in general no Rota-Baxter operator. To overcome this constraint we need the concept of free Rota-Baxter algebras as reviewed in Section 2.1.

In order to reproduce the $q-\mathrm{MZ}(\mathrm{S}) \mathrm{V}$ that are defined by iterated sums we define the summation operators

$$
P[f](x):=\sum_{n>0} f(x+n) \quad \text { and } \quad \bar{P}[f](x):=\sum_{n \geqslant 0} f(x+n) .
$$

Under certain regularity conditions on $f$ we can deduce that $P$ is a Rota-Baxter operator of weight 1 and $\bar{P}$ is one of weight -1 . For $f_{k}(t):=\frac{q^{k t}}{[t]_{q}^{k}}$ we regard the $\mathbb{Q}$-algebra

$$
\mathcal{A}_{q}:=\bigoplus_{k \in \mathbb{N}_{0}} \mathbb{Q} f_{k}(t)
$$


with a filtration given by $\mathcal{A}_{q, m}=\bigoplus_{k \geqslant m} \mathbb{Q} f_{k}(t)$. For f $:=\left(f_{1}, \ldots, f_{n}\right) \in \mathcal{A}_{q}^{n}$ we define the symbol $P_{\mathbf{f}}:=P\left[f_{1}\left[P\left[f_{2} \ldots P\left[f_{n}\right] \ldots\right]\right]\right]$. Since $\mathcal{A}_{q}$ is an iteratively summable subalgebra of level 1 of the algebra of continuous function $C_{q}(] 0, \infty[)$ on $] 0, \infty[$ in the parameter $q$, the MZV algebra is given by

$$
\mathfrak{A}_{q, 1}:=\left\{P_{\left(f_{1}, \ldots, f_{n}\right)}: f_{i} \in \mathcal{A}_{q}, i=1, \ldots, n, f_{n} \in \mathcal{A}_{q, 1}\right\} .
$$

The $M Z V$ algebra $\mathfrak{A}_{q, 1}$ is the algebra of $q$-MZVs if we employ the evaluation homomorphism $f \mapsto f(0)$. The algebra of $q$-MZSVs is obtained by replacing $P$ by $\bar{P}$ in $\mathfrak{A}_{q, 1}$ and employing the evaluation $f \mapsto f(1)$.

Theorem 6.2. Let $s \in \mathbb{N}$ and $\mathcal{A}$ be a unitary commutative $\mathbb{Q}$-algebra and $P$ a Rota-Baxter operator of weight $\lambda \in \mathbb{Q}^{\times}$. Then there is a unique sequence $\left(Q_{n}^{(\lambda)}\right)_{n \in \mathbb{N}}$ of polynomials with $Q_{n}^{(\lambda)} \in \mathbb{Q}\left[X_{1}, \ldots, X_{n}\right]$ and $\operatorname{deg}\left(Q_{n}^{(\lambda)}\right)=n$ such that

$$
P_{a^{s}}^{n}(1)=Q_{n}^{(\lambda)}\left(P\left(a^{s}\right), P\left(a^{2 s}\right), \ldots, P\left(a^{s n}\right)\right)
$$

for $a \in \mathcal{A}$ satisfying

(i) $Q_{0}^{(\lambda)}=1$ and $\nabla_{n} Q_{n}^{(\lambda)}=\sum_{k=1}^{n} \frac{(-\lambda)^{k-1}}{k} Q_{n-k}^{(\lambda)} \vec{e}_{k}$,

(ii) $Q_{n}^{(\lambda)}\left(\{0\}_{n}\right)=0$

for all $n \in \mathbb{N}$ in which $\nabla_{n}:=\sum_{k=1}^{n} \partial_{X_{k}} \vec{e}_{k}$ where $\vec{e}_{k}$ is the $k$-th canonical unit vector and $\{k\}_{n}$ denotes the $n$-tuple $(k, \ldots, k)$.

Corollary 6.3. For $k, n \in \mathbb{N}$ we have

$$
\zeta_{q}\left[\{k\}_{n}\right]=Q_{n}^{(1)}\left(\zeta_{q}[k], \zeta_{q}[2 k], \ldots, \zeta_{q}[n k]\right)
$$

and

$$
\zeta_{q}^{\star}\left[\{k\}_{n}\right]=Q_{n}^{(-1)}\left(\zeta_{q}^{\star}[k], \zeta_{q}^{\star}[2 k], \ldots, \zeta_{q}^{\star}[n k]\right),
$$

where $\left(Q_{n}^{(1)}\right)_{n \in \mathbb{N}}$ and $\left(Q_{n}^{(-1)}\right)_{n \in \mathbb{N}}$ are the unique sequences of polynomials in Theorem 6.2.

Proof. For the filtered algebra $\mathcal{A}_{q}$ we regard the corresponding free Rota-Baxter algebra $\left(\varpi_{\mathbb{Q}}\left(\mathcal{A}_{q}\right), P_{\mathcal{A}_{q}}\right)$ of weight 1 respectively -1 and we can apply Theorem 6.2. For $a:=f_{k}(t)=\frac{q^{k t}}{[t]_{q}^{k}} \in \mathcal{A}_{q}$, equation (14) is an algebraic relation in $\mathfrak{M}\left(\mathcal{A}_{q}\right)_{k}$. Using the evaluation $\nu: f \mapsto f(0)$ respectively $\nu: f \mapsto f(1)$, the relation reduces via $\nu \circ \mathfrak{P}_{k}$ to the claim in which $\mathfrak{P}_{k}\left(1 \otimes f_{1} \otimes f_{2} \otimes \cdots \otimes f_{n}\right):=P_{\left(f_{1}, \ldots, f_{n}\right)}$ respectively $\mathfrak{P}_{k}\left(1 \otimes f_{1} \otimes f_{2} \otimes \cdots \otimes f_{n}\right):=\bar{P}_{\left(f_{1}, \ldots, f_{n}\right)}$.

\section{Example 6.4.}

(a) For a Rota-Baxter operator of weight 1 we get the polynomials listed in Table 1. 
(b) The first three nontrivial identities for $q-\mathrm{MZVs}$ are

$$
\begin{aligned}
\zeta_{q}[s, s] & =\frac{1}{2} \zeta_{q}[s]^{2}-\frac{1}{2} \zeta_{q}[2 s], \\
\zeta_{q}[s, s, s] & =\frac{1}{6} \zeta_{q}[s]^{3}-\frac{1}{2} \zeta_{q}[s] \zeta_{q}[2 s]+\frac{1}{3} \zeta_{q}[3 s], \\
\zeta_{q}[s, s, s, s] & =\frac{1}{24} \zeta_{q}[s]^{4}-\frac{1}{4} \zeta_{q}[s]^{2} \zeta_{q}[2 s]+\frac{1}{3} \zeta_{q}[s] \zeta_{q}[3 s]+\frac{1}{8} \zeta_{q}[2 s]^{2}-\frac{1}{4} \zeta_{q}[4 s] .
\end{aligned}
$$

and for $q$-MZSV are

$$
\begin{aligned}
\zeta_{q}^{\star}[s, s] & =\frac{1}{2} \zeta_{q}^{\star}[s]^{2}+\frac{1}{2} \zeta_{q}^{\star}[2 s], \\
\zeta_{q}^{\star}[s, s, s] & =\frac{1}{6} \zeta_{q}^{\star}[s]^{3}+\frac{1}{2} \zeta_{q}^{\star}[s] \zeta_{q}^{\star}[2 s]+\frac{1}{3} \zeta_{q}^{\star}[3 s], \\
\zeta_{q}^{\star}[s, s, s, s] & =\frac{1}{24} \zeta_{q}^{\star}[s]^{4}+\frac{1}{4} \zeta_{q}^{\star}[s]^{2} \zeta_{q}^{\star}[2 s]+\frac{1}{3} \zeta_{q}^{\star}[s] \zeta_{q}^{\star}[3 s]+\frac{1}{8} \zeta_{q}^{\star}[2 s]^{2}+\frac{1}{4} \zeta_{q}^{\star}[4 s] .
\end{aligned}
$$

\begin{tabular}{|c|c|c|}
\hline$n$ & $p(n)$ & $Q_{n}^{(1)}$ \\
\hline 0 & 0 & 1 \\
1 & 1 & $X_{1}$ \\
2 & 2 & $\frac{1}{2} X_{1}^{2}-\frac{1}{2} X_{2}$ \\
3 & 3 & $\frac{1}{6} X_{1}^{3}-\frac{1}{2} X_{1} X_{2}+\frac{1}{3} X_{3}$ \\
4 & 5 & $\frac{1}{24} X_{1}^{4}-\frac{1}{4} X_{1}^{2} X_{2}+\frac{1}{3} X_{1} X_{3}+\frac{1}{8} X_{2}^{2}-\frac{1}{4} X_{4}$ \\
5 & 7 & $\frac{1}{120} X_{1}^{5}-\frac{1}{12} X_{1}^{3} X_{2}+\frac{1}{6} X_{1}^{2} X_{3}+\frac{1}{8} X_{1} X_{2}^{2}-\frac{1}{4} X_{1} X_{4}-\frac{1}{6} X_{2} X_{3}+\frac{1}{5} X_{5}$ \\
6 & 11 & $\frac{1}{720} X_{1}^{6}-\frac{1}{48} X_{1}^{4} X_{2}+\frac{1}{18} X_{1}^{3} X_{3}+\frac{1}{16} X_{1}^{2} X_{2}^{2}+\frac{1}{8} X_{1}^{2} X_{4}-\frac{1}{6} X_{1} X_{2} X_{3}-\frac{1}{48} X_{2}^{3}$ \\
& & $+\frac{1}{5} X_{1} X_{5}+\frac{1}{8} X_{2} X_{4}+\frac{1}{18} X_{3}^{2}-\frac{1}{6} X_{6}$ \\
\hline
\end{tabular}

Table 1. List of the polynomials $Q_{n}^{(1)}$ for a Rota-Baxter operator of weight 1 for $n=$ $1, \ldots, 6$, where $p(n)$ denotes the number of integer partitions of $n$.

Proposition 6.5. Let $s, n \in \mathbb{N}$. Then we have

$$
P_{a^{s}}^{n}(1)=(-\lambda)^{n} \sum_{r=1}^{n} \frac{(-\lambda)^{-r}}{r !} \sum_{\substack{n_{1}+\cdots+n_{r}=n \\ n_{1}, \ldots, n_{r} \geqslant 1}} \prod_{i=1}^{r} \frac{P\left(a^{s n_{i}}\right)}{n_{i}} .
$$

Proof. Let $s \in \mathbb{N}$. Now we consider

$$
f_{s}(z):=\sum_{k \geqslant 1} \frac{(-\lambda)^{k-1} z^{s k} P\left(a^{s k}\right)}{k} .
$$


From the Taylor expansion of the exponential function we can deduce

$$
\begin{aligned}
\frac{\partial^{s n}}{\partial z^{s n}} \exp \left(f_{s}(z)\right) & =\sum_{r \geqslant 0} \frac{1}{r !} \frac{\partial^{s n}}{\partial z^{s n}} f_{s}(z)^{r} \\
& =\sum_{r \geqslant 0} \frac{1}{r !} \sum_{\tilde{n}_{1}+\cdots+\tilde{n}_{r}=s n}\left(\begin{array}{c}
s n \\
\tilde{n}_{1}, \ldots, \tilde{n}_{r}
\end{array}\right) \prod_{i=1}^{r} \frac{\partial^{\tilde{n}_{i}}}{\partial z^{\tilde{n}_{i}}} f_{s}(z) .
\end{aligned}
$$

In the last step we used the generalized Leibniz rule. Now

$$
\left.\frac{\partial^{l}}{\partial z^{l}} f_{s}(z)\right|_{z=0}= \begin{cases}0 & \text { if } l \neq \equiv 0 \bmod s \text { or } l=0 \\ \frac{(-\lambda)^{l / s-1} l !}{l / s} P\left(a^{l}\right) & \text { if } l \equiv 0 \bmod s \text { and } l \neq 0\end{cases}
$$

implies

$$
\begin{aligned}
&\left.\frac{\partial^{s n}}{\partial z^{s n}} \exp \left(f_{s}(z)\right)\right|_{z=0} \\
&=\sum_{r \geqslant 0} \frac{1}{r !} \sum_{\substack{n_{1}+\cdots+n_{r}=n \\
n_{1}, \ldots, n_{r} \geqslant 1}}\left(\begin{array}{c}
s n \\
s n_{1}, \ldots, s n_{r}
\end{array}\right) \prod_{i=1}^{r} \frac{(-\lambda)^{n_{i}-1}\left(s n_{i}\right) !}{n_{i}} P\left(a^{s n_{i}}\right)
\end{aligned}
$$

and therefore we get

$$
\left.\frac{1}{(s n) !} \frac{\partial^{s n}}{\partial z^{s n}} \exp \left(f_{s}(z)\right)\right|_{z=0}=(-\lambda)^{n} \sum_{r=1}^{n} \frac{(-\lambda)^{-r}}{r !} \sum_{\substack{n_{1}+\cdots+n_{r}=n \\ n_{1}, \ldots, n_{r} \geqslant 1}} \prod_{i=1}^{r} \frac{P\left(a^{s n_{i}}\right)}{n_{i}} .
$$

Theorem 6.1 with $a$ substituted by $a^{s}$ implies the claim.

Proof of Theorem 6.2. Let $n, r \geqslant 1$. We introduce the following notation. Let $n_{1}+\cdots+n_{r}=n$ be an integer partition of $n$ with length $r$ and suppose that $n_{1} \geqslant \ldots \geqslant n_{r} \geqslant 1$. Therefore we have $1 \leqslant r \leqslant n$. Then we define

$$
a_{m}:=\#\left\{i \in\{1, \ldots, r\}: n_{i}=m\right\}
$$

for $1 \leqslant m \leqslant n$. Let $j \in\{1, \ldots n\}$ and $a_{j} \neq 0$. Then we can ensure that $a_{n-j+1}=\cdots=a_{n}=0$. Furthermore we denote by $\tilde{n}_{1}+\cdots+\tilde{n}_{\tilde{r}}=n-j$ the integer partition of $n-j$ with length $\tilde{r}$ arising from our old partition by deleting exactly one summand $j$. Now we have $0 \leqslant \tilde{r} \leqslant n-j$. We can conclude using the definition

$$
\tilde{a}_{m}:=\#\left\{i \in\{1, \ldots, \tilde{r}\}: \tilde{n}_{i}=m\right\}
$$

that $\tilde{a}_{i}=a_{i}$ for $i \neq j$ and $\tilde{a}_{j}=a_{j}-1$. If we substitute $P\left(a^{s n_{i}}\right)$ by the variable 
$X_{n_{i}}$ for $i \in\{1, \ldots, r\}$ we get

$$
\begin{aligned}
Q_{n}^{(\lambda)}\left(X_{1}, \ldots, X_{n}\right)= & (-\lambda)^{n} \sum_{r=1}^{n} \frac{(-\lambda)^{-r}}{r !} \sum_{\substack{n_{1}+\cdots+n_{r}=n \\
n_{1}, \ldots, n_{r} \geqslant 1}} \prod_{\substack{i=1 \\
n_{i}}}^{X_{n_{i}}} \\
= & (-\lambda)^{n} \sum_{r=1}^{n}(-\lambda)^{-r} \sum_{\substack{n_{1}+\cdots+n_{r}=n \\
n_{1} \geqslant \cdots \geqslant n_{r} \geqslant 1, a_{j}=0}} \frac{1}{a_{1} ! \cdots a_{n} !} \prod_{i=1}^{n} \frac{X_{i}^{a_{i}}}{i_{i}^{a}} \\
& +\left(1-\delta_{n}(j)\right)(-\lambda)^{n} \sum_{r=1}^{n}(-\lambda)^{-r} \sum_{\substack{n+\cdots+n_{r}=n \\
n_{1} \geqslant \cdots \geqslant n_{r} \geqslant 1, a_{j} \neq 0}} \frac{1}{a_{1} ! \cdots a_{n} !} \prod_{i=1}^{n} \frac{X_{i}^{a_{i}}}{i^{a_{i}}} \\
& +\frac{(-\lambda)^{n-1}}{n} X_{n} \delta_{n}(j),
\end{aligned}
$$

where $\delta_{n}(j)$ denotes Kronecker's delta. Now we determine $\partial_{X_{j}} Q_{n}^{(\lambda)}\left(X_{1}, \ldots, X_{n}\right)$. The first term in the last equation vanishes since it does not depend on $X_{j}$. Now we get

$$
\begin{aligned}
& \partial_{X_{j}} Q_{n}^{(\lambda)}\left(X_{1}, \ldots, X_{n}\right) \\
& =\left(1-\delta_{n}(j)\right)(-\lambda)^{n} \sum_{r=1}^{n}(-\lambda)^{-r} \sum_{\substack{n_{1}+\cdots+n_{r}=n \\
n_{1} \geqslant \cdots \geqslant n_{r} \geqslant 1, a_{j} \neq 0}} \frac{1}{a_{1} ! \cdots\left(a_{j}-1\right) ! \cdots a_{n} !} \frac{X_{j}^{a_{j}-1}}{j^{a_{j}}} \prod_{\substack{i=1 \\
i \neq j}}^{n} \frac{X_{i}^{a_{i}}}{i^{a_{i}}} \\
& +\frac{(-\lambda)^{n-1}}{n} \delta_{n}(j) \\
& =\left(1-\delta_{n}(j)\right) \frac{(-\lambda)^{j-1}}{j}(-\lambda)^{n-j} \sum_{r=1}^{n-j}(-\lambda)^{-r} \sum_{\substack{\tilde{n}_{1}+\cdots+\tilde{n}_{r}=n-j \\
\tilde{n}_{1} \geqslant \cdots \geqslant \tilde{n}_{r} \geqslant 1}} \frac{1}{\tilde{a}_{1} ! \cdots \tilde{a}_{j} ! \cdots \tilde{a}_{n-j} !} \prod_{i=1}^{n-j} \frac{X_{i}^{\tilde{a}_{i}}}{i^{\tilde{a}_{i}}} \\
& +\frac{(-\lambda)^{n-1}}{n} \delta_{n}(j) \\
& =\frac{(-\lambda)^{j-1}}{j} Q_{n-j}^{(\lambda)}\left(X_{1}, \ldots, X_{n-j}\right) .
\end{aligned}
$$

Therefore condition (i) is verified. (ii) is an immediate consequence of Proposition 6.5. Uniqueness can be shown by induction on $n$ using condition (i).

Acknowledgement. I am very grateful to Andreas Knauf (Erlangen) who proposed me to work on the subject of MZV and for his comments on this work. Further I am much obliged to Kurusch Ebrahimi-Fard (Madrid) and Dominique Manchon (Clermont-Ferrand) who explained me their papers [4, 3] and for discussion and comments on this article. Finally I thank Henrik Bachmann (Hamburg) for implementing and sharing his Pari GP code for the $q$-analogues studied in this paper. 
Note added in proof. The author was informed that J. Zhao published a preprint (arXiv:1412.8044) in December 2014 that includes parts of the results provided in this work.

\section{Appendix A. Comments on [5]}

In this appendix we just recall the main results of [5]. It has already been mentioned by Castillo Medina, Ebrahimi-Fard and Manchon in [4] that there are some problems with the display of the results concerning Theorem 3. However it is possible to correct the calculations in the proof. Let $(\mathcal{A}, P)$ be a Rota-Baxter algebra of weight $\lambda$. Then for $a, b, c \in \mathbb{N}_{0}$ the operator $T(a, b, c): \mathcal{A} \times \mathcal{A} \rightarrow \mathcal{A}$ is given by

$$
T(a, b, c):=P^{c}\left[P^{a}[\cdot] \cdot P^{b}[\cdot]\right]
$$

Then we have the following results:

Theorem A.1 ([5]). Let $a, b>1$ and $c \geqslant 0$ be integers. The following identity holds in any Rota-Baxter algebra of weight 0 :

$$
\begin{aligned}
T(a, b, c)= & \sum_{i=0}^{b-1}\left(\begin{array}{c}
a+i-1 \\
a-1
\end{array}\right) T(0, b-i, a+c+i) \\
& +\sum_{i=0}^{a-1}\left(\begin{array}{c}
b+i-1 \\
b-1
\end{array}\right) T(a-i, 0, b+c+i)
\end{aligned}
$$

Theorem A.2 ([5],[4]). Let $a, b \geqslant 1$ and $c \geqslant 0$ be integers. The following identity holds in any Rota-Baxter algebra of weight $\lambda$ :

$$
\begin{aligned}
T(a, b, c)= & \sum_{(i, j) \in D_{1}} \lambda^{a+b-i-j}\left(\begin{array}{c}
j-1 \\
i+j-b-1, j-a, a+b-i-j
\end{array}\right) T(0, i, c+j) \\
& +\sum_{(i, j) \in D_{2}} \lambda^{a+b-i-j}\left(\begin{array}{c}
j-1 \\
i+j-b, j-a, a+b-i-j-1
\end{array}\right) T(0, i, c+j) \\
& +\sum_{(i, j) \in D_{3}} \lambda^{a+b-i-j}\left(\begin{array}{c}
j-1 \\
j-b, i+j-a-1, a+b-i-j
\end{array}\right) T(i, 0, c+j) \\
& +\sum_{(i, j) \in D_{4}} \lambda^{a+b-i-j}\left(\begin{array}{c}
j-1 \\
j-b, i+j-a, a+b-i-j-1
\end{array}\right) T(i, 0, c+j) \\
& +\sum_{j \in D_{5}} \lambda^{a+b-j}\left(\begin{array}{c}
j-1 \\
j-b, j-a, a+b-j-1
\end{array}\right) T(0,0, c+j),
\end{aligned}
$$


where

$$
\begin{aligned}
& D_{1}:=\left\{(i, j) \in \mathbb{N}^{2}: 1 \leqslant i \leqslant b, a \leqslant j, b-i+1 \leqslant j, j \leqslant a+b-i\right\}, \\
& D_{2}:=\left\{(i, j) \in \mathbb{N}^{2}: 1 \leqslant i \leqslant b-1, a \leqslant j, b-i \leqslant j, j \leqslant a+b-i-1\right\}, \\
& D_{3}:=\left\{(i, j) \in \mathbb{N}^{2}: 1 \leqslant i \leqslant a, a-i+1 \leqslant j, b \leqslant j, j \leqslant a+b-i\right\}, \\
& D_{4}:=\left\{(i, j) \in \mathbb{N}^{2}: 1 \leqslant i \leqslant a-1, a-i \leqslant j, b \leqslant j, j \leqslant a+b-i-1\right\}, \\
& D_{5}:=\{j \in \mathbb{N}: a \leqslant j, b \leqslant j, j \leqslant a+b-1\} .
\end{aligned}
$$

\section{References}

[1] D. Bradley, A q-analog of Euler's decomposition formula for the double zeta function, Int. J. Math. Math. Sci. 21 (2005), 3453-3458.

[2] D. Bradley, Multiple q-zeta values, J. Algebra (2005), 752-798.

[3] J. Castillo Medina, K. Ebrahimi-Fard, D. Manchon, Unfolding the double shuffle structure of q-multiple zeta values, Bull. Aust. Math. Soc. 91(3) (2015), 368-388.

[4] J. Castillo Medina, K. Ebrahimi-Fard, D. Manchon, On Euler's decomposition formula for qMZVs, Ramanujan J. 37(2) (2015), 365-389.

[5] R. Diaz, M. Paez, An Identity in Rota-Baxter Algebras, Sem. Lothar. Combin. 57 (B57b) (2007).

[6] K. Ebrahimi-Fard, L. Guo, Multiple zeta values and Rota-Baxter algebras, Integers 8(2) (2006).

[7] L. Guo, An Introduction to Rota-Baxter Algebra, volume IV of Surveys of Modern Mathematics.

[8] L. Guo, W. Keigher, Baxter algebras and shuffle products, Adv. Math (2000), $117-149$.

[9] L. Guo, W. Keigher, On free Baxter algebras: completions and the internal construction, Adv. Math. (2000), 101-127.

[10] M. Hoffman, Multiple harmonic series, Pacific Journal of Mathematics 152(2) (1992), 275-290.

[11] M. Hoffman, Quasi-shuffle products, J. Algebraic Combin. 11(1) (2000), 4968 .

[12] K. Ihara, M. Kaneko, D. Zagier, Derivation and double shuffle relations for multiple zeta values, Compos. Math. 142 (2006).

[13] Y. Ohno, J. Okuda, W. Zudilin, Cyclic $q-M Z S V$ sum, J. Number Theory 132(1) (2012), 144-155.

[14] G.-C. Rota, D. Smith, Fluctuation theory and Baxter algebras, Instituto Nazionale di Alta Matematica IX (1972), 179-201.

[15] K.-G. Schlesinger, Some remarks on q-deformed multiple polylogarithms, arXiv:math/0111022, 2001.

[16] J. Singer, On Bradley's q-MZVs and a Generalized Euler Decomposition Formula, submitted,

[17] Y. Takeyama, The Algebra of a q-Analogue of Multiple Harmonic Series, SIGMA 9. 
[18] D. Zagier, Values of zeta functions and their applications, in First European Congress of Mathematics, volume 120, 497-512. Birkhäuser-Verlag, Basel, 1994.

[19] J. Zhao, Multiple q-zeta functions and multiple q-polylogarithms, Ramanujan J. 14(2) (2007), 189-221.

[20] W. Zudilin, Algebraic relations for multiple zeta values, Uspekhi Mat. Nauk 58, 2003.

Address: Johannes Singer: Department Mathematik, Friedrich-Alexander-Universität ErlangenNürnberg, Cauerstraße 11, 91058 Erlangen, Germany.

E-mail: singer@math.fau.de

Received: 3 October 2014; revised: 27 January 2015 